\title{
Total Synthesis of Leupyrrin B1: a Potent Inhibitor of Human Leukocyte Elastase
}

Sebastian Thiede, ${ }^{\dagger}$ Paul R. Wosniok, ${ }^{\dagger}$ Daniel Herkommer, ${ }^{\dagger}$ Anna-Christina Schulz-Fincke, ${ }^{\dagger}$ Michael Gütschow, ${ }^{\dagger}$ and Dirk Menche ${ }^{*} \dagger$

†Kekulé-Institut für Organische Chemie und Biochemie, Universität Bonn, Gerhard-Domagk-Str. 1, D-53121 Bonn, Germany and Pharmazeutisches Institut, Universität Bonn, An der Immenburg 4, D-53121 Bonn, Germany

dirk.menche@uni-bonn.de

\section{Supporting Information}

\section{Table of Contents:}

1 GENERAL METHODS

2 EXPERIMENTAL PROCEDURES AND CHARACTERIZATION DATA.......................................................

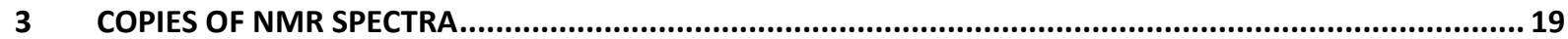

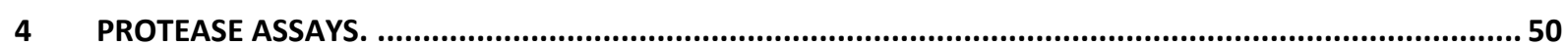




\section{General Methods}

All reactions with dry solvents were performed under an atmosphere of argon in flame-dried glassware which had been cooled under argon unless stated otherwise. All flasks were equipped with rubber septa and reactants were handled using standard Schlenk techniques.

Temperatures above room temperature $\left(20^{\circ} \mathrm{C}\right)$ refer to oil bath temperatures which were controlled by a temperature modulator. For cooling, the following baths were used: acetone/dry ice $\left(-78^{\circ} \mathrm{C}\right), \mathrm{H}_{2} \mathrm{O} /$ ice $\left(0^{\circ} \mathrm{C}\right)$ for other temperatures below $0{ }^{\circ} \mathrm{C}$ a Huber TC100EF-NR cooler was used.

All reagents were purchased from commercial suppliers (Sigma-Aldrich, TCI, AlfaAesar, Strem) in the highest grade available and used without further purification unless otherwise stated.

Anhydrous solvents (THF, $\mathrm{Et}_{2} \mathrm{O}$, toluene, and $\mathrm{CH}_{2} \mathrm{Cl}_{2}$ ) were obtained from a solvent drying system MB SPS-800 (MBraun) and stored over molecular sieves or purchased over molecular sieves (dioxane, DMF, DMSO).

Reactions were monitored via TLC on silica gel $60 \mathrm{~F}_{254}$ precoated plates $\left(0.2 \mathrm{~mm} \mathrm{SiO}_{2}\right.$, Machery-Nagel) and visualized using UV light and/or staining with a solution of CAM (1.0 g $\mathrm{Ce}\left(\mathrm{SO}_{4}\right)_{2}, 2.5 \mathrm{~g}\left(\mathrm{NH}_{4}\right)_{6} \mathrm{Mo}_{7} \mathrm{O}_{24}, 8 \mathrm{~mL}$ conc. $\mathrm{H}_{2} \mathrm{SO}_{4}$ in $\left.100 \mathrm{~mL} \mathrm{H}_{2} \mathrm{O}\right)$ or $\mathrm{KMnO}_{4}(1.5 \mathrm{~g}$ $\mathrm{KMnO}_{4}, 10 \mathrm{~g} \mathrm{~K}_{2} \mathrm{CO}_{3}, 1.25 \mathrm{~mL} 10 \% \mathrm{NaOH}$ in $200 \mathrm{~mL} \mathrm{H} \mathrm{H}_{2} \mathrm{O}$ ) or 4-anisaldehyde (3.7 mL 4anisaldehyde, $1.5 \mathrm{~mL} \mathrm{HOAc,} 5 \mathrm{~mL} \mathrm{H}_{2} \mathrm{SO}_{4}$ in $135 \mathrm{~mL}$ abs. EtOH) respectively, and subsequent heating.

For column chromatography, silica gel (pore size $60 \AA$, 40-63 $\mu \mathrm{m}$ ) obtained from Aldrich or Merck was used and the size of the column was adjusted to the recommendations by Still et $a l .{ }^{1}$ Compounds were eluated using the stated mixtures under a positive pressure of air. Solvents were destilled prior to use.

Optical rotations were measured with a Perkin Elmer 241 or 341 polarimeter in a $10 \mathrm{~mm}$ cuvette and are uncorrected.

${ }^{1} \mathrm{H}$ - and ${ }^{13} \mathrm{C}-\mathrm{NMR}$ spectra were recorded on Bruker AV-300, DRX-300, DPX-300, DPX-400, DRX-500, Avance-I-500, Avance-III-500, Avance-III-600, and Avance-III-700 spectrometers with ${ }^{13} \mathrm{C}$ operating frequencies of $75,100,125,150$ and $175 \mathrm{Mhz}$ respectively. Spectra were measured at room temperature unless stated otherwise. Chemical shifts $(\delta)$ are reported in ppm relative to tetramethylsilane $(\delta=0.00 \mathrm{ppm})$ and the spectra were calibrated to the

\footnotetext{
${ }^{1}$ Still, W. C.; Kahn, M.; Mitra A. J. Org. Chem. 1978, 43, 2923.
} 
residual signal of undeuterated solvents. ${ }^{2}$ Data for ${ }^{1} \mathrm{H}-\mathrm{NMR}$ spectra are reported as follows: chemical shift (multiplicity, coupling constants in Hertz, number of hydrogens). Abbreviations are as follows: $\mathrm{s}$ (singlet), $\mathrm{d}$ (doublet), $\mathrm{t}$ (triplet), $\mathrm{q}$ (quartet), quint (quintet), $\mathrm{spt}$ (septet), m (multiplet), br. (broad).

Mass spectra (MS) and high-resolution-mass spectra (HRMS) were recorded at the Department of Organic Chemistry in Bonn on the following mass spectrometers: Bruker Daltonics micrOTOF-Q, Bruker Daltonics autoflex II TOF/TOF, Thermo Fisher Scientific Orbitrap XL and a Thermo Finnigan MAT 95 XL.

The HPLC analytical and preparative analyses were performed by using a system of the company "Knauer Wissenschaftliche Geräte GmbH": Smartline series consisting of a twochannel degasser, two pumps S-1800 (100 mL pump head), an injection assistant 6000 with a feed pump S-100, a mixing chamber Smartmix 350, a UV-detector S-2550 and a fraction valves (16 port). The system was controlled by Chromgate software version 3.3.2. All solvents were purchased from the central chemical store of the University of Bonn in HPLC grade.

\section{Sources of compounds.}

Compounds 10, 11, 13, 19, 20, 21, and 26 were obtained as previously described. ${ }^{3}$

List of abbreviations:

\begin{tabular}{|c|c|}
\hline Boc & tert-butyloxycarbonyl \\
\hline DAST & diethylaminosulfur trifluoride \\
\hline DMAP & 4-(N,N-dimethylamino)pyridine \\
\hline HATU & $\begin{array}{l}O \text {-(7-azabenzotriazol-1-yl)- } N, N, N, N \cdot \text {-tetramethyluronium } \\
\text { hexafluorophosphate }\end{array}$ \\
\hline MS & molecular sieve \\
\hline $\mathrm{rt}$ & room temperature \\
\hline TASF & tris(dimethylamino)sulfonium difluorotrimethylsilicate \\
\hline TBAF & tetra-n-butylammoniumfluoride \\
\hline TBS & tert-butyldimethylsilyl \\
\hline TCBC & 2,4,6-trichlorbenzoylchloride \\
\hline Teoc & 2-(trimethylsilyl)ethoxycarbonyl \\
\hline
\end{tabular}

\footnotetext{
${ }^{2}$ Fulmer, G. R.; Miller, A. J. M.; Sherden, N. H.; Gottlieb, H. E.; Nudelman, A.; Stoltz, B. M.; Bercaw, J. E.; Goldberg, K. I. Organometallics 2010, 29, 2176.

${ }^{3}$ Herkommer, D.; Thiede, S.; Wosniok, P. R.; Dreisigacker, S.; Tian, M.; Debnar, T.; Irschik H.; Menche, D. J. Am. Chem. Soc. 2015, 137, 4086.
} 


\section{Experimental Procedures and Characterization Data}

Methyl 4-methyl-2-oxopentanoate (7) ${ }^{4}$

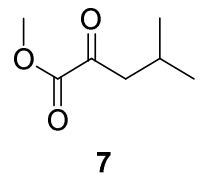

$\mathrm{C}_{7} \mathrm{H}_{12} \mathrm{O}_{3}, \mathrm{M}=144.2 \mathrm{~g} / \mathrm{mol}$

To a solution of 4-methyl-2-oxopentanoic acid (3.4 g, $26.1 \mathrm{mmol}, 1.0$ equiv) in methanol $(12 \mathrm{~mL})$ and 2,2-dimethoxypropane $(48 \mathrm{~mL})$ is added chlorotrimethylsilane $(0.38 \mathrm{~mL}$, $2.99 \mathrm{mmol}, 0.1$ equiv). The reaction is stirred at room temperature overnight. The reaction is concentrated under reduced pressure $\left(40^{\circ} \mathrm{C}, 30 \mathrm{mbar}\right)$ to give 7 as colorless oil (3.69 $\mathrm{g}$, $25.6 \mathrm{mmol}, 98 \%)$.

${ }^{1}$ H-NMR $\left(300 \mathrm{MHz}, \mathrm{CDCl}_{3}\right) \delta[\mathrm{ppm}]=0.96(\mathrm{~d}, J=6.7 \mathrm{~Hz}, 6 \mathrm{H}), 2.19$ (tspt, $J=6.8$, $6.7 \mathrm{~Hz}, 1 \mathrm{H}), 2.71(\mathrm{~d}, J=6.8 \mathrm{~Hz}, 2 \mathrm{H}), 3.85(\mathrm{~s}, 3 \mathrm{H}) ;{ }^{13} \mathbf{C}-\mathbf{N M R}\left(75 \mathrm{MHz}, \mathrm{CDCl}_{3}\right) \delta[\mathrm{ppm}]$ $=22.6(2 \mathrm{C}), 24.3,48.1,53.0,161.9,194.1$; HRMS (ESI+): calculated for $\mathrm{C}_{7} \mathrm{H}_{12} \mathrm{O}_{3} \mathrm{Na}^{+}$ $[\mathrm{M}+\mathrm{Na}]^{+}: 167.0679$, found: 167.0675 .

\section{Methyl 2-(diethoxyphosphoryl)-4-methylpentanoate (9) ${ }^{5}$}

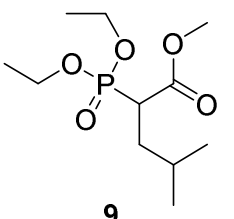

$$
\mathrm{C}_{11} \mathrm{H}_{23} \mathrm{O}_{5} \mathrm{P}, \mathrm{M}=266.2 \mathrm{~g} / \mathrm{mol}
$$

Methyl 2-bromo-4-methylpentanoate (3.74 g, 17.9 mmol, 1.0 equiv, synthesis: see ref 6) and triethyl phosphite $\left(5.95 \mathrm{~g}, 35.8 \mathrm{mmol}, 2.0\right.$ equiv) are heated to $150{ }^{\circ} \mathrm{C}$. The reaction temperature is increased to $200{ }^{\circ} \mathrm{C}$ over $6 \mathrm{~h}$ and resulting bromoethane (b. p. $38^{\circ} \mathrm{C}$ ) is

\footnotetext{
${ }^{4}$ Genin, M. J.; Agejas-Chicharro, F. J.; Bueno Melendo, A. B.; Manninen, P. R.; Warshawsky, A. M. Compounds And Methods For Modulating FXR. PCT/US2008/069719, 16 July, 2007.

${ }^{5}$ Donald, D. K.; Hann, M. M.; Saunders, J.; Wadsworth, H. J. U.S. Patent 703,973, Februar 21, 1985.

${ }^{6}$ Géant, P.-Y.; Martínez, J.; Salom-Roig, X. J. Eur. J. Org. Chem. 2011, 1300.
} 
collected with an air condenser. The residue is destilled under high vacuum affording 9 as colorless oil (4.10 g, $17.9 \mathrm{mmol}, 86 \%)$.

${ }^{1}$ H-NMR $\left(400 \mathrm{MHz}, \mathrm{CDCl}_{3}\right) \delta[\mathrm{ppm}]=0.87(\mathrm{~d}, J=6.3 \mathrm{~Hz}, 3 \mathrm{H}), 0.90(\mathrm{~d}, J=6.3 \mathrm{~Hz}, 3 \mathrm{H})$, $1.32(\mathrm{t}, J=7.1 \mathrm{~Hz}, 6 \mathrm{H}), 1.47-1.64(\mathrm{~m}, 2 \mathrm{H}), 1.92-2.04(\mathrm{~m}, 1 \mathrm{H}), 3.04(\mathrm{ddd}, J=23.2,12.2$, $4.0 \mathrm{~Hz}, 1 \mathrm{H}), 3.73(\mathrm{~s}, 3 \mathrm{H}), 4.08-4.19(\mathrm{~m}, 4 \mathrm{H}) ;{ }^{13} \mathbf{C}-\mathbf{N M R}\left(100 \mathrm{MHz}, \mathrm{CDCl}_{3}\right) \delta[\mathrm{ppm}]=$ 16.5 (d, $J=6 \mathrm{~Hz}, 1 \mathrm{C}), 16.5$ (d, $J=6 \mathrm{~Hz}, 1 \mathrm{C}), 21.3,23.0,27.1$ (d, $J=15 \mathrm{~Hz}, 1 \mathrm{C}), 35.7$ (d, $J=5 \mathrm{~Hz}, 1 \mathrm{C}), 44.0(\mathrm{~d}, J=131 \mathrm{~Hz}, 1 \mathrm{C}), 52.5,62.8$ (d, $J=7 \mathrm{~Hz}, 1 \mathrm{C}), 62.9$ (d, $J=7 \mathrm{~Hz}$, 1C), $170.1(\mathrm{~d}, J=5 \mathrm{~Hz}, 1 \mathrm{C})$; HRMS (ESI+): calculated for $\mathrm{C}_{11} \mathrm{H}_{23} \mathrm{NaO}_{5} \mathrm{P}^{+}[\mathrm{M}+\mathrm{Na}]^{+}$: 289.1175, found: 289.1182 .

\section{4-(tert-Butyl) 1-methyl 2-isobutylfumarate (12)}

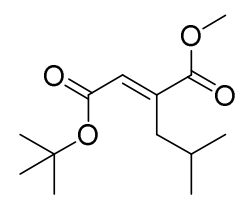

12

$\mathrm{C}_{13} \mathrm{H}_{22} \mathrm{O}_{4}, \mathrm{M}=242.3 \mathrm{~g} / \mathrm{mol}$

To a solution of (tert-butoxycarbonylmethylene)triphenylphosphorane (444 $\mathrm{mg}$, $1.18 \mathrm{mmol}, 1.0$ equiv) in dry DCM (5 mL) is added 7 (170 mg, $1.18 \mathrm{mmol}, 1.0$ equiv) and stirred under argon at room temperature for $2 \mathrm{~h}$. The reaction is concentrated under reduced pressure and the crude product is purified by column chromatography $\left(\mathrm{SiO}_{2}\right.$, cyclohexane/ethyl acetate $=30 / 1)$ to give 12 (4-(tert-butyl) 1-methyl 2-isobutylfumarate) as colorless oil (209 mg, $0.861 \mathrm{mmol}, 73 \%)$. Also the Z-product (4-(tert-butyl) 1-methyl 2-isobutylmaleate) is obtained (68.6 $\mathrm{mg}, 0.283 \mathrm{mmol}, 24 \%)$ as colorless oil.

4-(tert-butyl) 1-methyl 2-isobutylfumarate (12):

$\mathbf{R}_{\mathbf{f}}=0.55$ (cyclohexane/ethyl acetate $\left.=9 / 1\right) ;{ }^{1} \mathbf{H}-\mathbf{N M R}\left(500 \mathrm{MHz}, \mathrm{CDCl}_{3}\right) \delta[\mathrm{ppm}]=0.89$ (d, $J=6.7 \mathrm{~Hz}, 6 \mathrm{H}), 1.49$ (s, 9H), 1.80 (tspt, $J=6.8,6.7 \mathrm{~Hz}, 1 \mathrm{H}), 2.69$ (d, $J=6.8 \mathrm{~Hz}, 2 \mathrm{H})$, $3.77(\mathrm{~s}, 3 \mathrm{H}), 6.69(\mathrm{~s}, 1 \mathrm{H}) ;{ }^{13} \mathrm{C}-\mathbf{N M R}\left(75 \mathrm{MHz}, \mathrm{CDCl}_{3}\right) \delta[\mathrm{ppm}]=22.4(2 \mathrm{C}), 28.3(3 \mathrm{C})$, 28.7, 35.8, 52.5, 81.5, 129.5, 145.3, 165.3, 168.2; HRMS (ESI+): calculated for $\mathrm{C}_{13} \mathrm{H}_{22} \mathrm{O}_{4} \mathrm{Na}^{+}[\mathrm{M}+\mathrm{Na}]^{+}:$265.1410, found: 265.1409. 
4-(tert-butyl) 1-methyl 2-isobutylmaleate:

$\mathbf{R}_{\mathbf{f}}=0.38$ (cyclohexane/ethyl acetate $\left.=9 / 1\right) ;{ }^{1} \mathbf{H}-\mathbf{N M R}\left(500 \mathrm{MHz}, \mathrm{CDCl}_{3}\right) \delta[\mathrm{ppm}]=0.93$ (d, $J=6.7 \mathrm{~Hz}, 6 \mathrm{H}), 1.46$ (s, 9H), 1.78 (tspt, $J=7.2,6.7 \mathrm{~Hz}, 1 \mathrm{H}), 2.18$ (dd, $J=7.3,1.3 \mathrm{~Hz}$, 2H), $3.80(\mathrm{~s}, 3 \mathrm{H}), 5.71(\mathrm{t}, J=1.3 \mathrm{~Hz}, 1 \mathrm{H}) ;{ }^{13} \mathbf{C}-\mathbf{N M R}\left(75 \mathrm{MHz}, \mathrm{CDCl}_{3}\right) \delta[\mathrm{ppm}]=22.4$ (2C), 26.6 (3C), 28.2, 44.1, 52.2, 81.3, 123.1, 147.6, 164.3, 169.6; HRMS (ESI+): calculated for $\mathrm{C}_{13} \mathrm{H}_{22} \mathrm{O}_{4} \mathrm{Na}^{+}[\mathrm{M}+\mathrm{Na}]^{+}: 265.1410$, found: 265.1409 .

(E)-2-(2-(tert-Butoxy)-2-oxoethylidene)-4-methylpentanoic acid (14)

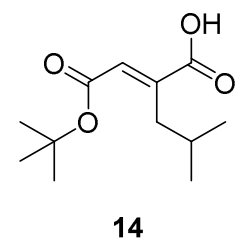

$$
\mathrm{C}_{12} \mathrm{H}_{20} \mathrm{O}_{4}, \mathrm{M}=228.3 \mathrm{~g} / \mathrm{mol}
$$

To a solution of 4-(tert-Butyl) 1-methyl 2-isobutylfumarate (700 mg, $2.88 \mathrm{mmol}, 1.0$ equiv) in $7 \mathrm{~mL}$ THF and $7 \mathrm{~mL} \mathrm{H}_{2} \mathrm{O}$ is added $\mathrm{LiOH} \cdot \mathrm{H}_{2} \mathrm{O}(242 \mathrm{mg}, 2.0$ equiv) and the resulting mixture is refluxed at $95{ }^{\circ} \mathrm{C}$ for $60 \mathrm{~min}$. After cooling to ambient temperature, ether $(70 \mathrm{~mL})$, water $(70 \mathrm{~mL})$ and sat. $\mathrm{NaHCO}_{3}$ solution $(10 \mathrm{~mL})$ are added consecutively. After phase separation, the aqueous phase is acidified with $1 \mathrm{M} \mathrm{HCl}$ and extracted with ether $(3 \times 100 \mathrm{~mL})$. The combined organic extracts were dried with $\mathrm{MgSO}_{4}$, filtered and concentrated under reduced pressure. The crude product is purified by column chromatography $\left(\mathrm{SiO}_{2}\right.$, cyclohexane/ethyl acetate/acetic acid $\left.=9 / 1,5 / 0,2\right)$ to give the title compound as colorless solid (572 $\mathrm{mg}, 2.51 \mathrm{mmol}, 87 \%)$.

$\mathbf{R}_{\mathbf{f}}=0.40$ (cyclohexane/ethyl acetate/acetic acid =9/2/0,2) ${ }^{\mathbf{1}} \mathbf{H}-\mathbf{N M R}\left(400 \mathrm{MHz}, \mathrm{CD}_{2} \mathrm{Cl}_{2}\right.$ ) $\delta[\mathrm{ppm}]=0.91(\mathrm{~d}, J=6.6 \mathrm{~Hz}, 6 \mathrm{H}), 1.50(\mathrm{~s}, 9 \mathrm{H}), 1.85(\mathrm{tspt}, J=7.3,6.6 \mathrm{~Hz}, 1 \mathrm{H}), 2.66(\mathrm{~d}$, $J=7.3 \mathrm{~Hz}, 2 \mathrm{H}), 6.81(\mathrm{~s}, 1 \mathrm{H}) ;{ }^{13} \mathbf{C}-\mathbf{N M R}\left(100 \mathrm{MHz}, \mathrm{CD}_{2} \mathrm{Cl}_{2}\right) \delta[\mathrm{ppm}]=22.5(2 \mathrm{C}), 28.2$ (3C), 28.7, 35.5, 81.8, 131.5, 144.1, 165.1, 173.1; HRMS (ESI-): calculated for $\mathrm{C}_{12} \mathrm{H}_{19} \mathrm{O}_{4}{ }^{-}$ [M-H] : 227.1289, found: 227.1289. 
4-(tert-Butyl) 1-(((4R,5R)-4-((Z)-((S,Z)-2-isobutyl-4-(5-methoxypentan-2-ylidene)dihydrofuran-3(2H)-ylidene)methyl)-2,2-dimethyl-3-((2-(trimethylsilyl)ethoxy)carbonyl)oxazolidin-5-yl)methyl) 2-isobutylfumarate (15)

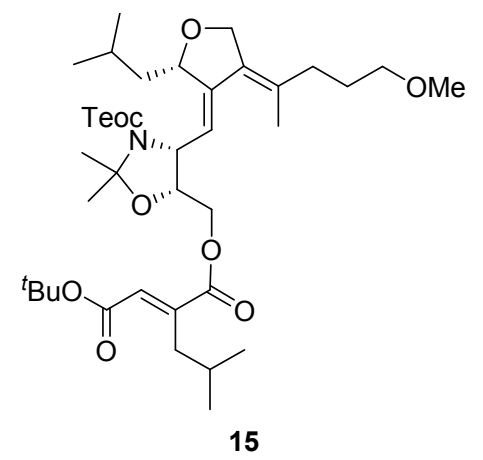

$\mathrm{C}_{39} \mathrm{H}_{67} \mathrm{NO} 9 \mathrm{Si}, \mathrm{M}=722.0 \mathrm{~g} / \mathrm{mol}$

To a solution of acid $14\left(107 \mathrm{mg}, 469 \mu \mathrm{mol}, 2.0\right.$ equiv) in dry $\mathrm{CH}_{2} \mathrm{Cl}_{2}(4.7 \mathrm{~mL})$ was added $\mathrm{NEt}_{3}(260 \mu \mathrm{L}, 1.88 \mathrm{mmol}, 8.0$ equiv), MNBA (162 mg, $469 \mu \mathrm{mol}, 2.0$ equiv) and DMAP (43 mg, $352 \mu \mathrm{mol}, 1.5$ equiv) and the resulting mixture was stirred at room temperature for 10 minutes. Alcohol 13 (120 mg, $234 \mu \mathrm{mol}, 1.0$ equiv) was dissolved in dry $\mathrm{CH}_{2} \mathrm{Cl}_{2}(5.8 \mathrm{~mL})$, added to the reaction mixture and stirred for 60 minutes at room temperature. The reaction was quenched by the addition of saturated $\mathrm{NaHCO}_{3}$ solution $(10 \mathrm{~mL})$. The organic phase was separated and the aqueous phase was extracted with $\mathrm{CH}_{2} \mathrm{Cl}_{2}(3 \times 10 \mathrm{~mL})$. The combined organic layers were dried over $\mathrm{MgSO}_{4}$, filtered and the solvent was removed in vacuo. The crude product was purified by column chromatography ( $\mathrm{SiO}_{2}$, cyclohexane/EtOAc, 9:1) yielding 15 (163 mg, $\left.226 \mathrm{mmol}, 96 \%\right)$ as a colorless oil.

$\mathbf{R}_{\mathbf{f}}=0.33($ cyclohexane/EtOAc $=9 / 1) ;[\alpha]_{D}^{20}=-124.9\left(\mathrm{c}=1.0, \mathrm{CH}_{2} \mathrm{Cl}_{2}\right) ;{ }^{1} \mathbf{H}-\mathbf{N M R}$ $(700 \mathrm{MHz}, 339 \mathrm{~K}$, acetone-d6): $\delta[\mathrm{ppm}]=0.05(\mathrm{~s}, 9 \mathrm{H}), 0.89(\mathrm{~d}, J=6.7 \mathrm{~Hz}, 6 \mathrm{H}), 0.96(\mathrm{~d}$, $J=6.9 \mathrm{~Hz}, 3 \mathrm{H}), 0.98(\mathrm{~d}, J=6.7 \mathrm{~Hz}, 3 \mathrm{H}), 0.99-1.06(\mathrm{~m}, 2 \mathrm{H}), 1.42-1.47$ (m, 1H), 1.50 (s, $9 \mathrm{H}), 1.54-1.60(\mathrm{~m}, 1 \mathrm{H}), 1.57(\mathrm{~s}, 3 \mathrm{H}), 1.65(\mathrm{~s}, 3 \mathrm{H}), 1.66-1.71(\mathrm{~m}, 2 \mathrm{H}), 1.80-1.86(\mathrm{~m}, 1 \mathrm{H})$, 1.81-1.88 (m, 1H), $1.91(\mathrm{~s}, 3 \mathrm{H}), 2.01-2.10(\mathrm{~m}, 2 \mathrm{H}), 2.69(\mathrm{dd}, J=7.2,1.4 \mathrm{~Hz}, 2 \mathrm{H}), 3.26(\mathrm{~s}$, $3 \mathrm{H}), 3.31(\mathrm{t}, J=6.1 \mathrm{~Hz}, 2 \mathrm{H}), 4.11-4.17(\mathrm{~m}, 1 \mathrm{H}), 4.16-4.21(\mathrm{~m}, 1 \mathrm{H}), 4.26(\mathrm{~d}, J=5.8 \mathrm{~Hz}$, 2H), $4.36(\mathrm{~d}, J=11.8 \mathrm{~Hz}, 1 \mathrm{H}), 4.45(\mathrm{~d}, J=12.0 \mathrm{~Hz}, 1 \mathrm{H}), 4.55$ (q, $J=5.7 \mathrm{~Hz}, 1 \mathrm{H}), 4.66$ $(\mathrm{dd}, J=9.8,5.3 \mathrm{~Hz}, 1 \mathrm{H}), 4.81(\mathrm{~d}, J=11.2 \mathrm{~Hz}, 1 \mathrm{H}), 5.42$ (d, $J=10.1 \mathrm{~Hz}, 1 \mathrm{H}), 6.70$ (s, 1H); ${ }^{13}$ C-NMR (125 MHz, $323 \mathrm{~K}$, acetone-d6) $\delta[\mathrm{ppm}]=-1.3$ (3C), 19.5, 20.0, 22.3, 
22.8, 22.9, 24.4, 24.7, 26.0, 28.1, 28.3, 28.5 (3C), 29.7, 34.6, 36.6, 42.6, 58.6, 59.4, 63.5, 64.4, 69.5, 72.6, 76.0, 79.5, 82.0, 94.9, 120.0, 130.2, 131.8, 132.4, 145.0, 145.8, 153.2, 165.7, 167.6; HRMS (ESI+): $\mathrm{C}_{39} \mathrm{H}_{67} \mathrm{NNaO}_{9} \mathrm{Si}[\mathrm{M}+\mathrm{Na}]^{+}:$744.4477, found 744.4471.

\section{1-((2R,3R,Z)-3-Amino-2-hydroxy-4-((S,Z)-2-isobutyl-4-(5-methoxypentan-2-} ylidene)dihydrofuran-3(2H)-ylidene)butyl) 4-(tert-butyl) 2-isobutylfumarate (5)

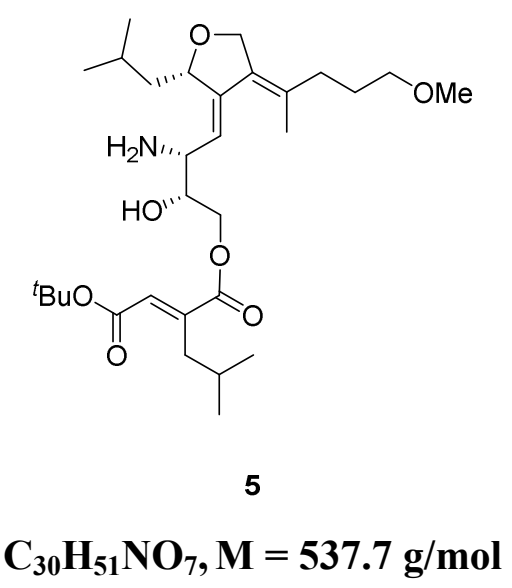

TAS-F (90\%, $22.0 \mathrm{mg}, 72.0 \mu \mathrm{mol}, 2.0$ equiv) was dissolved in dry MeCN (220 $\mu \mathrm{L})$ and added dropwise to a solution of $15(26.0 \mathrm{mg}, 36.0 \mu \mathrm{mol}, 1.0$ equiv) in dry $\operatorname{MeCN}(360 \mu \mathrm{L})$ at $0{ }^{\circ} \mathrm{C}$. After 10 minutes the ice bath was removed and the reaction mixture was slowly warmed to room temperature. After stirring for 50 minutes at this temperature the reaction was diluted with EtOAc $(10 \mathrm{~mL})$ and quenched by the addition of saturated $\mathrm{NH}_{4} \mathrm{Cl}$ solution $(10 \mathrm{~mL})$. The organic phase was separated and the aqueous phase was extracted with $\mathrm{Et}_{2} \mathrm{O}(2 \times 10 \mathrm{~mL})$ and EtOAc $(10 \mathrm{~mL})$. The combined organic layers were dried over $\mathrm{MgSO}_{4}$, filtered and concentrated in vacuo. The crude product was filtered over a small pad of silica, eluating with cyclohexane/EtOAc/i $\mathrm{PrNH}_{2} 60 / 40 / 3$. The solvent was removed in vacuo yielding 5 (19.4 mg, $36.0 \mu \mathrm{mol}, 100 \%)$ as yellow oil which was directly used without further purification. NMR analysis showed a purity of $>70 \%$. (NOTE: slow column chromatography or purification by preparative TLC gave rise to small quantities of the more polar byproduct S12 by migration of the acyl group to the amine). 


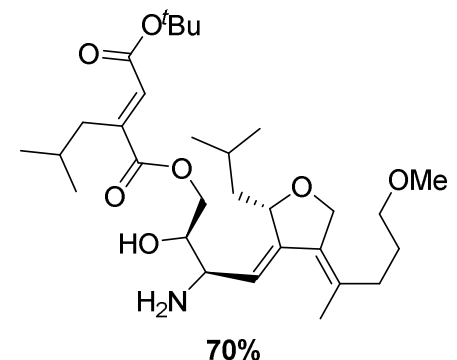

25

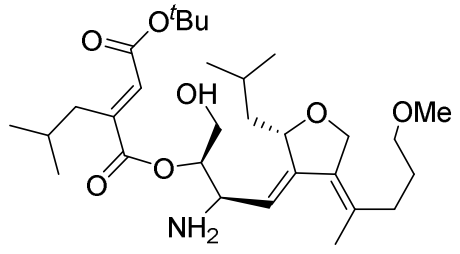

$30 \%$

S11

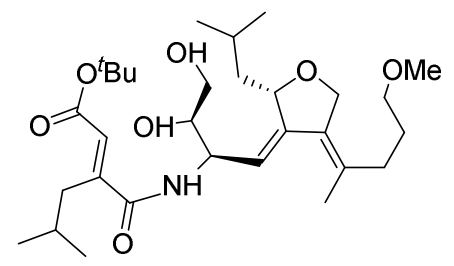

S12

$\mathbf{R}_{\mathbf{f}}\left(\right.$ cyclohexane $/$ EtOAc/ $\left.i \mathrm{PrNH}_{2}, 50 / 50 / 3\right)=0.24$.

$3 H$-[1,2,3] triazolo[4,5-b]pyridin-3-yl $1 H$-pyrrole-2-carboxylate (17)

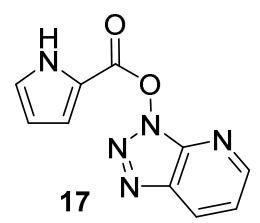

$$
\mathrm{C}_{10} \mathrm{H}_{7} \mathrm{~N}_{5} \mathrm{O}_{2}, \mathrm{M}=229.2 \mathrm{~g} / \mathrm{mol}
$$

To a solution of $1 H$-pyrrole-2-carboxylic acid $(22.2 \mathrm{mg}, 200 \mu \mathrm{mol}, 1.0$ equiv) in dry $\mathrm{MeCN}(5.0 \mathrm{~mL})$ at $-30{ }^{\circ} \mathrm{C} \mathrm{NEt}_{3}(56 \mu \mathrm{L}, 400 \mu \mathrm{mol}, 2.0$ equiv) and HATU $(91.2 \mathrm{mg}$, $240 \mu$ mol, 1.2 equiv) were added. The resulting solution was stirred for 90 minutes at $30{ }^{\circ} \mathrm{C}$ before it was concentrated under reduced pressure. Purification by preparative TLC ( $\mathrm{SiO}_{2}$, cyclohexane/EtOAc, 50:50) gave $17(41.3 \mathrm{mg}, 180 \mu \mathrm{mol}, 90 \%)$ as a colorless oil. $\mathbf{R}_{\mathbf{f}}=0.51$ (cyclohexane/EtOAc $\left.=1 / 1\right) ;{ }^{1} \mathbf{H}-\mathbf{N M R}\left(300 \mathrm{MHz}, \mathrm{CD}_{2} \mathrm{Cl}_{2}\right): \delta[\mathrm{ppm}]=6.46(\mathrm{dt}$, $J=4.0,2.5 \mathrm{~Hz}, 1 \mathrm{H}), 7.26(\mathrm{td}, J=2.8,1.4 \mathrm{~Hz}, 1 \mathrm{H}), 7.39$ (ddd, $J=4.0,2.5,1.4 \mathrm{~Hz}, 1 \mathrm{H})$, $7.47(\mathrm{dd}, J=8.4,4.5 \mathrm{~Hz}, 1 \mathrm{H}), 8.45(\mathrm{dd}, J=8.4,1.4 \mathrm{~Hz}, 1 \mathrm{H}), 8.73(\mathrm{dd}, J=4.5,1.4 \mathrm{~Hz}$, 1H), 9.79 (br. s, $1 \mathrm{H}) ;{ }^{13} \mathbf{C}-\mathbf{N M R}\left(75 \mathrm{MHz}, \mathrm{CD}_{2} \mathrm{Cl}_{2}\right) \delta[\mathrm{ppm}]=112.4,116.8,120.5,121.5$, $127.8,130.1,135.6,141.4,152.4,157.0 ; \mathbf{H R M S}(\mathrm{ESI}+): \mathbf{C}_{\mathbf{1 0}} \mathbf{H}_{7} \mathbf{N}_{\mathbf{5}} \mathrm{NaO}_{2}{ }^{+}[\mathrm{M}+\mathrm{Na}]^{+}:$ 252.0492 , found 252.0497 . 
$3 H-[1,2,3]$ Triazolo[4,5-b]pyridin-3-yl 5-((E)-3-((2S,3R,4R)-3-acetoxy-4-((tertbutyldimethylsilyl)oxy)methyl)-4-methyl-5-oxotetrahydrofuran-2-yl)but-2-en-1-yl)$1 \mathrm{H}$-pyrrole-2-carboxylate (22)

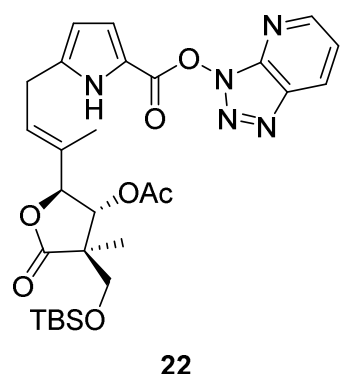

\section{$\mathrm{C}_{28} \mathrm{H}_{37} \mathrm{~N}_{5} \mathrm{O}_{7} \mathrm{Si}, \mathrm{M}=583.7 \mathrm{~g} / \mathrm{mol}$}

To a solution of acid 4 (90.0 mg, $193 \mu \mathrm{mol}, 1.0$ equiv) in dry $\mathrm{MeCN}(5.0 \mathrm{~mL})$ at $-30{ }^{\circ} \mathrm{C}$ $\mathrm{NEt}_{3}(54 \mu \mathrm{L}, 387 \mu \mathrm{mol}, 2.0$ equiv) and HATU (88.2 mg, $232 \mu \mathrm{mol}, 1.2$ equiv) were added. The resulting solution was stirred for 90 minutes at $-30{ }^{\circ} \mathrm{C}$ before it was concentrated under reduced pressure. Purification by column chromatography $\left(\mathrm{SiO}_{2}\right.$, cyclohexane/EtOAc, 2:1) gave $22(96.0 \mathrm{mg}, 164 \mu \mathrm{mol}, 85 \%)$ as a colorless oil.

$\mathbf{R}_{\mathbf{f}}=0.56($ cyclohexane $/$ EtOAc $=1 / 1) ;[\alpha]_{D}^{20}=-5.9\left(\mathrm{c}=1.0, \mathrm{CH}_{2} \mathrm{Cl}_{2}\right) ;{ }^{1} \mathbf{H}-\mathbf{N M R}$ $\left(400 \mathrm{MHz}, \mathrm{CD}_{2} \mathrm{Cl}_{2}\right): \delta[\mathrm{ppm}]=0.05(\mathrm{~s}, 3 \mathrm{H}), 0.06(\mathrm{~s}, 3 \mathrm{H}), 0.87(\mathrm{~s}, 9 \mathrm{H}), 1.11(\mathrm{~s}, 3 \mathrm{H}), 1.80$ (s, 3H), 2.06 (s, 3H), 3.50 (dd, $J=16.9,8.9 \mathrm{~Hz}, 1 \mathrm{H}), 3.52$ (d, $J=9.7 \mathrm{~Hz}, 1 \mathrm{H}), 3.61$ (dd, $J=16.9,8.9 \mathrm{~Hz}, 1 \mathrm{H}), 3.82(\mathrm{~d}, J=9.7 \mathrm{~Hz}, 1 \mathrm{H}), 4.57$ (d, $J=7.9 \mathrm{~Hz}, 1 \mathrm{H}), 5.70-5.73$ (m, $1 \mathrm{H}), 5.73-5.76(\mathrm{~m}, 1 \mathrm{H}), 6.21(\mathrm{dd}, J=3.8,2.8 \mathrm{~Hz}, 1 \mathrm{H}), 7.33(\mathrm{dd}, J=3.9,2.6 \mathrm{~Hz}, 1 \mathrm{H})$, $7.46(\mathrm{dd}, J=8.4,4.5 \mathrm{~Hz}, 1 \mathrm{H}), 8.44(\mathrm{dd}, J=8.5,1.3 \mathrm{~Hz}, 1 \mathrm{H}), 8.71(\mathrm{dd}, J=4.6,1.3 \mathrm{~Hz}$, 1H), 9.62 (br. s, $1 \mathrm{H}) ;{ }^{13} \mathbf{C}-\mathbf{N M R}\left(125 \mathrm{MHz}, \mathrm{CD}_{2} \mathrm{Cl}_{2}\right) \delta[\mathrm{ppm}]=-5.4,-5.4,11.5,14.3$, 18.7, 21.0, 26.1 (3C), 27.1, 50.0, 65.8, 72.7, 84.6, 110.9, 115.7, 121.4, 121.6, 126.1, $130.0,135.3,135.6,141.2,141.5,152.4,156.7,171.4,177.1$; HRMS (ESI+): $\mathrm{C}_{28} \mathrm{H}_{37} \mathrm{~N}_{5} \mathrm{NaO}_{7} \mathrm{Si}^{+}[\mathrm{M}+\mathrm{Na}]^{+}: 606.2354$, found 606.2359 . 
1-((2R,3R,Z)-3-(5-((E)-3-((2S,3R,4R)-3-Acetoxy-4-((tert-

butyldimethylsilyl)oxy)methyl)-4-methyl-5-oxotetrahydrofuran-2-yl)but-2-en-1-yl)$1 H$-pyrrole-2-carboxamido)-2-hydroxy-4-((R,Z)-2-isobutyl-4-(5-methoxypentan-2ylidene)dihydrofuran-3(2H)-ylidene)butyl) 4-(tert-butyl) 2-isobutylfumarate (3)

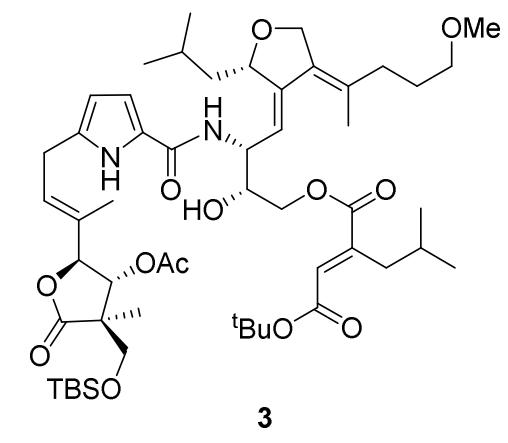

$$
\mathrm{C}_{53} \mathrm{H}_{84} \mathrm{~N}_{2} \mathrm{O}_{13} \mathrm{Si}, \mathrm{M}=985.3 \mathrm{~g} / \mathrm{mol}
$$

To a solution of activated acid $22(17.0 \mathrm{mg}, 29.1 \mu \mathrm{mol}, 1.0$ equiv) in dry MeCN (700 $\mu \mathrm{L})$ at $0{ }^{\circ} \mathrm{C} \mathrm{NEt}_{3}(8.1 \mu \mathrm{L}, 58.3 \mu \mathrm{mol}, 2.0$ equiv) and $\mathbf{5}(15.7 \mathrm{mg}, 35.0 \mu \mathrm{mol}, 1.2$ equiv) in dry $\mathrm{MeCN}(700 \mu \mathrm{L})$ were added consecutively. The resulting solution was stirred for 1 hour at $0{ }^{\circ} \mathrm{C}$ before it was diluted with EtOAc $(5 \mathrm{~mL})$ and concentrated under reduced pressure. Purification by preparative TLC $\left(\mathrm{SiO}_{2}\right.$, cyclohexane/EtOAc, 1:1) gave 3 (26.1 mg, $26.5 \mu \mathrm{mol}, 91 \%$ ) as a colorless gum.

$\mathbf{R}_{\boldsymbol{f}}=0.54$ (cyclohexane/EtOAc $\left.=1 / 1\right) ; \quad[\alpha]_{D}^{20}=-87.6\left(c=1.0\right.$ in $\left.\mathrm{CH}_{2} \mathrm{Cl}_{2}\right) ;{ }^{1} \mathbf{H}-\mathbf{N M R}$ $\left(400 \mathrm{MHz}, \mathrm{CD}_{2} \mathrm{Cl}_{2}\right): \delta[\mathrm{ppm}]=0.06(\mathrm{~s}, 3 \mathrm{H}), 0.08(\mathrm{~s}, 3 \mathrm{H}), 0.85-0.89(\mathrm{~m}, 6 \mathrm{H}), 0.88(\mathrm{~s}, 9 \mathrm{H})$, 0.89-0.92 (m, 6H), 1.08 (s, 3H), 1.40-1.46 (m, 1H), 1.49 (s, 9H), 1.61-1.70 (m, 1H), 1.64$1.72(\mathrm{~m}, 2 \mathrm{H}), 1.75(\mathrm{~s}, 3 \mathrm{H}), 1.75-1.81(\mathrm{~m}, 1 \mathrm{H}), 1.77-1.84(\mathrm{~m}, 1 \mathrm{H}), 1.93(\mathrm{~s}, 3 \mathrm{H}), 2.01-2.06$ (m, 2H), $2.08(\mathrm{~s}, 3 \mathrm{H}), 2.66(\mathrm{~d}, J=7.3 \mathrm{~Hz}, 2 \mathrm{H}), 3.29(\mathrm{~s}, 3 \mathrm{H}), 3.32(\mathrm{t}, J=6.3 \mathrm{~Hz}, 2 \mathrm{H})$, 3.41-3.46 (m, 3H), $3.56(\mathrm{~d}, J=9.7 \mathrm{~Hz}, 1 \mathrm{H}), 3.82(\mathrm{~d}, J=9.5 \mathrm{~Hz}, 1 \mathrm{H}), 4.16-4.26(\mathrm{~m}, 2 \mathrm{H})$, 4.36-4.51 (m, 2H), $4.56(\mathrm{~d}, J=7.7 \mathrm{~Hz}, 1 \mathrm{H}), 4.71$ (ddd, $J=9.9,7.6,3.8 \mathrm{~Hz}, 1 \mathrm{H}), 4.84$ (dt, $J=10.5,2.0 \mathrm{~Hz}, 1 \mathrm{H}), 5.65-5.71(\mathrm{~m}, 1 \mathrm{H}), 5.68-5.74(\mathrm{~m}, 1 \mathrm{H}), 5.70-5.76(\mathrm{~m}, 1 \mathrm{H}), 5.94(\mathrm{t}$, $J=3.2 \mathrm{~Hz}, 1 \mathrm{H}), 6.15(\mathrm{~d}, J=7.7 \mathrm{~Hz}, 1 \mathrm{H}), 6.53(\mathrm{dd}, J=3.6,2.6 \mathrm{~Hz}, 1 \mathrm{H}), 6.69(\mathrm{~s}, 1 \mathrm{H})$, 9.18 (br. s., $1 \mathrm{H}) ;{ }^{13} \mathrm{C}-\mathbf{N M R}\left(125 \mathrm{MHz}, \mathrm{CD}_{2} \mathrm{Cl}_{2}\right): \delta[\mathrm{ppm}]=-5.4,-5.4,11.4,14.2,18.7$, 20.5, 20.9, 22.0, 22.7, 22.7, 24.0, 25.6, 26.1 (3C), 26.9, 27.9, 28.4 (3C), 29.2, 34.7, 36.3, $43.3,50.1,52.8,58.8,66.0,67.2,69.7,72.4,72.6,73.0,80.0,82.0,84.5,108.2,108.4$, $110.8,117.0,125.1,127.6,130.2,130.8,133.5,135.1,145.1,147.3,161.0,165.5$, 168.1, 
170.8, 177.3; HRMS (ESI+): calculated for $\mathrm{C}_{53} \mathrm{H}_{84} \mathrm{~N}_{2} \mathrm{NaO}_{13} \mathrm{Si}^{+}[\mathrm{M}+\mathrm{Na}]^{+}: 1007.5635$, found: 1007.5616 .

1-(((4R,5S)-2-(5-((E)-3-((2S,3R,4R)-3-Acetoxy-4-(((tert-butyldimethylsilyl)oxy)methyl)-4-methyl-5-oxotetrahydrofuran-2-yl)but-2-en-1-yl)-1 H-pyrrol-2-yl)-4-((Z)((S,Z)-2-isobutyl-4-(5-methoxypentan-2-ylidene)dihydrofuran-3(2H)-ylidene)methyl)-4,5-dihydrooxazol-5-yl)methyl) 4-(tert-butyl) (S)-2-isobutylfumarate (23)

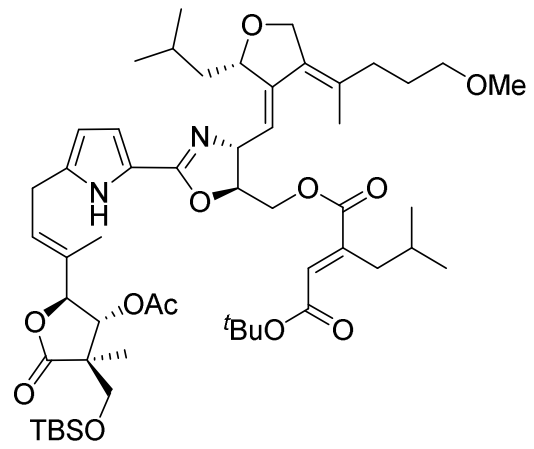

23

\section{$\mathrm{C}_{53} \mathrm{H}_{82} \mathrm{~N}_{2} \mathrm{O}_{12} \mathrm{Si}, \mathrm{M}=967.3 \mathrm{~g} / \mathrm{mol}$}

To a solution of $3\left(20.0 \mathrm{mg}, 20.3 \mu \mathrm{mol}, 1.0\right.$ equiv) in dry $\mathrm{CH}_{2} \mathrm{Cl}_{2}(400 \mu \mathrm{L})$ at $-78{ }^{\circ} \mathrm{C}$ a solution of DAST ( $4 \mu \mathrm{L}, 30.5 \mu \mathrm{mol}, 1.5$ equiv) in dry $\mathrm{CH}_{2} \mathrm{Cl}_{2}(120 \mu \mathrm{L})$ was added. The resulting solution was stirred for 40 minutes at $-78^{\circ} \mathrm{C}$ before anhydrous $\mathrm{K}_{2} \mathrm{CO}_{3}(5.6 \mathrm{mg}$, $40.6 \mu \mathrm{mol}, 1.5$ equiv) was added. The mixture was allowed to warm up to $-10{ }^{\circ} \mathrm{C}$ before it was quenched by addition of a saturated solution of $\mathrm{NaHCO}_{3}(1.5 \mathrm{~mL})$. The layers were separated and it was extracted with $\mathrm{Et}_{2} \mathrm{O}(3 \times 2 \mathrm{~mL})$. The organic layers were combined, dried over $\mathrm{MgSO}_{4}$ and concentrated in vacuo. Purification by preparative TLC $\left(\mathrm{SiO}_{2}\right.$, cyclohexane/EtOAc, 3:2) gave 23 (17.6 mg, $18.2 \mu \mathrm{mol}, 90 \%)$ as a white foam.

$\mathbf{R}_{\boldsymbol{f}}=0.54$ (cyclohexane/EtOAc $\left.=2 / 1\right) ;[\alpha]_{D}^{20}=-54.2\left(c=1.0\right.$ in $\left.\mathrm{CH}_{2} \mathrm{Cl}_{2}\right) ;{ }^{1} \mathbf{H}-\mathbf{N M R}$ $\left(400 \mathrm{MHz}, \mathrm{CD}_{2} \mathrm{Cl}_{2}\right): \delta[\mathrm{ppm}]=0.06(\mathrm{~s}, 3 \mathrm{H}), 0.07(\mathrm{~s}, 3 \mathrm{H}), 0.82-0.86(\mathrm{~m}, 6 \mathrm{H}), 0.88(\mathrm{~s}, 9 \mathrm{H})$, $0.94(\mathrm{~d}, J=6.6 \mathrm{~Hz}, 3 \mathrm{H}), 0.95$ (d, $J=6.6 \mathrm{~Hz}, 3 \mathrm{H}), 1.07$ (s, 3H), 1.33 (ddd, $J=14.2,9.5$, $2.7 \mathrm{~Hz}, 1 \mathrm{H}), 1.48$ (s, 9H), 1.57 (ddd, $J=14.4,10.6,4.3 \mathrm{~Hz}, 1 \mathrm{H}), 1.64-1.72$ (m, 2H), 1.75 (s, 3H), 1.76-1.80 (m, 1H), 1.81-1.88 (m, 1H), $1.90(\mathrm{~s}, 3 \mathrm{H}), 1.98-2.06(\mathrm{~m}, 2 \mathrm{H}), 2.07$ (s, $3 \mathrm{H}), 2.63(\mathrm{~d}, J=7.3 \mathrm{~Hz}, 2 \mathrm{H}), 3.29(\mathrm{~s}, 3 \mathrm{H}), 3.31$ (t, $J=6.3 \mathrm{~Hz}, 2 \mathrm{H}), 3.38-3.48(\mathrm{~m}, 2 \mathrm{H})$, $3.56(\mathrm{~d}, J=9.5 \mathrm{~Hz}, 1 \mathrm{H}), 3.81(\mathrm{~d}, J=9.7 \mathrm{~Hz}, 1 \mathrm{H}), 4.29-4.36(\mathrm{~m}, 1 \mathrm{H}), 4.39-4.45(\mathrm{~m}, 1 \mathrm{H})$, 
4.42-4.47 (m, 1H), 4.46-4.51 (m, 1H), 4.50-4.56 (m, 1H), 4.51-4.58 (m, 1H), 4.53-4.58 $(\mathrm{m}, 1 \mathrm{H}), 4.83-4.89(\mathrm{~m}, 1 \mathrm{H}), 5.46(\mathrm{~d}, J=8.3 \mathrm{~Hz}, 1 \mathrm{H}), 5.68-5.72(\mathrm{~m}, 1 \mathrm{H}) 5.72(\mathrm{~d}, J=$ $8.1 \mathrm{~Hz}, 1 \mathrm{H}), 5.95$ (d, $J=3.5 \mathrm{~Hz}, 1 \mathrm{H}), 6.60$ (d, $J=3.6 \mathrm{~Hz}, 1 \mathrm{H}), 6.67$ (s, 1H), 9.03 (br. s., $1 \mathrm{H}) ;{ }^{13} \mathbf{C}-\mathbf{N M R}\left(100 \mathrm{MHz}, \mathrm{CD}_{2} \mathrm{Cl}_{2}\right): \delta[\mathrm{ppm}]=-5.4,-5.4,11.4,14.1,18.7,20.5,21.0$, 22.0, 22.6, 22.6, 24.1, 25.5, 26.1 (3C), 27.0, 27.9, 28.4 (3C), 29.1, 34.7, 36.2, 44.0, 50.2, $58.8,65.6,66.0,68.5,69.8,72.4,72.5,79.8,81.9,83.4,84.4,108.3,113.9,119.5,122.3$, $127.7,130.5,130.7,133.2,133.2,135.3,144.8,144.8,158.3,165.4,167.7,170.6,177.3$; HRMS (ESI+): calculated for $\mathrm{C}_{53} \mathrm{H}_{83} \mathrm{~N}_{2} \mathrm{O}_{12} \mathrm{Si}^{+}[\mathrm{M}+\mathrm{H}]^{+}: 967.5710$, found: 967.5701 .

(E)-3-((((4R,5S)-2-(5-((E)-3-((2S,3R,4R)-3-Acetoxy-4-(((tert-butyldimethylsilyl)oxy)methyl)-4-methyl-5-oxotetrahydrofuran-2-yl)but-2-en-1-yl)-1 $H$-pyrrol-2-yl)-4-((Z)((S,Z)-2-isobutyl-4-(5-methoxypentan-2-ylidene)dihydrofuran-3(2H)ylidene)methyl)-4,5-dihydrooxazol-5-yl)methoxy)carbonyl)-5-methylhex-2-enoic acid (23a)

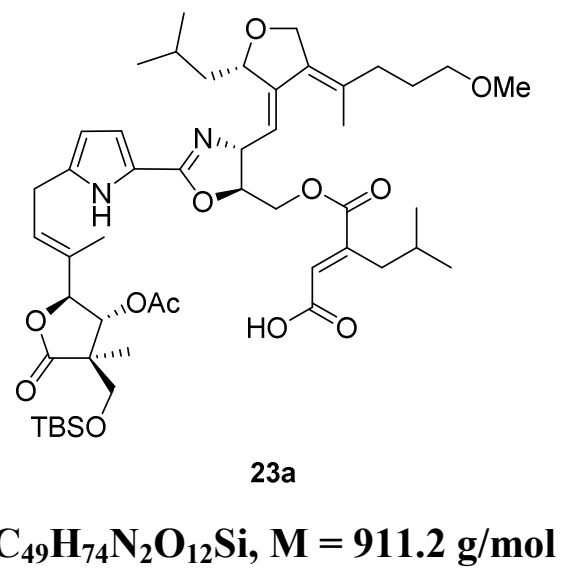

To a solution of 23 (10.0 mg, $10.3 \mu \mathrm{mol}, 1.0$ equiv) and 2,6-lutidine (36.1 $\mu \mathrm{L}, 310 \mu \mathrm{mol}$, 30 equiv) in dry $\mathrm{CH}_{2} \mathrm{Cl}_{2}(230 \mu \mathrm{L})$ at $0{ }^{\circ} \mathrm{C}$ TMSOTf $(28 \mu \mathrm{L}, 155 \mu \mathrm{mol}, 15$ equiv) was added. The resulting solution was stirred for 2 hours at $0{ }^{\circ} \mathrm{C}$ before it was allowed to warm up to room temperature. After further 30 minutes the reaction was diluted with EtOAc $(2 \mathrm{~mL})$ and quenched by addition of a saturated solution of $\mathrm{NH}_{4} \mathrm{Cl}(2 \mathrm{~mL})$. The layers were separated and the aqueous layer was extracted with EtOAc $(3 \times 2 \mathrm{~mL})$. The organic layers were combined, dried over $\mathrm{MgSO}_{4}$, concentrated in vacuo and the crude product was purified by preparative TLC $\left(\mathrm{SiO}_{2}\right.$, cyclohexane/EtOAc/HOAc, 5:5:0.2). Co- 
evaporation with toluene to remove residual acetic acid yielded $23 \mathbf{a}(8.5 \mathrm{mg}, 9.33 \mu \mathrm{mol}$, $90 \%$ ) as a white solid.

$\mathbf{R}_{\boldsymbol{f}}=0.66($ cyclohexane $/$ EtOAc/HOAc $=50 / 50 / 2) ;[\alpha]_{D}^{20}=-49.0(c=0.7$ in $\mathrm{MeOH}) ;{ }^{1} \mathbf{H}-$ NMR $\left(700 \mathrm{MHz}, \mathrm{CD}_{3} \mathrm{OD}\right) \delta[\mathrm{ppm}]=0.09(\mathrm{~s}, 3 \mathrm{H}), 0.10(\mathrm{~s}, 3 \mathrm{H}), 0.81(\mathrm{t}, J=6.6 \mathrm{~Hz}, 6 \mathrm{H})$, $0.91(\mathrm{~s}, 9 \mathrm{H}), 0.99$ (t, $J=6.8 \mathrm{~Hz}, 6 \mathrm{H}), 1.11(\mathrm{~s}, 3 \mathrm{H}), 1.37-1.43(\mathrm{~m}, 1 \mathrm{H}), 1.59-1.65(\mathrm{~m}, 1 \mathrm{H})$, $1.67-1.77(\mathrm{~m}, 2 \mathrm{H}), 1.72-1.80(\mathrm{~m}, 1 \mathrm{H}), 1.78(\mathrm{~s}, 3 \mathrm{H}), 1.85-1.91(\mathrm{~m}, 1 \mathrm{H}), 1.97(\mathrm{~s}, 3 \mathrm{H})$, $2.08(\mathrm{~s}, 3 \mathrm{H}), \quad 2.07-2.18(\mathrm{~m}, 2 \mathrm{H}), 2.67(\mathrm{~d}, J=6.0 \mathrm{~Hz}, 2 \mathrm{H}), 3.34(\mathrm{~s}, 3 \mathrm{H}), 3.38(\mathrm{t}, J=$ $6.0 \mathrm{~Hz}, 2 \mathrm{H}), 3.47(\mathrm{~d}, J=7.1 \mathrm{~Hz}, 2 \mathrm{H}), 3.62(\mathrm{~d}, J=9.5 \mathrm{~Hz}, 1 \mathrm{H}), 3.83(\mathrm{~d}, J=9.5 \mathrm{~Hz}, 1 \mathrm{H})$, 4.45-4.51 (m, 1H), 4.47-4.52 (m, 1H), $4.55(\mathrm{~d}, J=12.1 \mathrm{~Hz}, 1 \mathrm{H}), 4.63(\mathrm{dd}, J=12.5,2.2$ $\mathrm{Hz}, 1 \mathrm{H}), 4.67-4.71(\mathrm{~m}, 1 \mathrm{H}), 4.69-4.73(\mathrm{~m}, 1 \mathrm{H}), 4.74(\mathrm{~d}, J=8.2 \mathrm{~Hz}, 1 \mathrm{H}), 4.96(\mathrm{~d}, J=10.5$ $\mathrm{Hz}, 1 \mathrm{H}), 5.58(\mathrm{~d}, J=9.5 \mathrm{~Hz}, 1 \mathrm{H}), 5.81(\mathrm{~d}, J=8.2 \mathrm{~Hz}, 1 \mathrm{H}), 5.82(\mathrm{~d}, J=7.5 \mathrm{~Hz}, 1 \mathrm{H}), 5.97$ $(\mathrm{d}, J=3.2 \mathrm{~Hz}, 1 \mathrm{H}), 6.76-6.79(\mathrm{~m}, 1 \mathrm{H}), 6.77-6.80(\mathrm{~m}, 1 \mathrm{H}) ;{ }^{13} \mathbf{C}-\mathbf{N M R}\left(175 \mathrm{MHz}, \mathrm{CD}_{3} \mathrm{OD}\right)$ $\delta[\mathrm{ppm}]=-5.5,-5.5,11.3,13.9,19.1,20.3,20.5,22.1,22.7,22.7,24.1,26.1,26.3,27.1$, 28.3 (3C), 29.6, 35.0, 36.9, 44.8, 51.2, 58.8, 65.6, 66.5, 68.0, 70.3, 72.9, 73.1, 80.5, 84.4, $85.2,109.0,116.3,118.5,122.9,129.0,130.6,131.2,133.0,134.2,137.5,145.5,145.8$, $161.0,168.4,169.0,171.5,179.1$;

HRMS (ESI+): calculated for $\mathrm{C}_{49} \mathrm{H}_{75} \mathrm{~N}_{2} \mathrm{O}_{12} \mathrm{Si}^{+}[\mathrm{M}+\mathrm{H}]^{+}$: 911.5084 , found: 911.5087 . 
(E)-3-((((4R,5S)-2-(5-((E)-3-((2S,3R,4R)-4-(((tert-Butyldimethylsilyloxy)methyl)-3hydroxy-4-methyl-5-oxotetrahydrofuran-2-yl)but-2-en-1-yl)-1H-pyrrol-2-yl)-4-((Z)((S,Z)-2-isobutyl-4-(5-methoxypentan-2-ylidene)dihydrofuran-3(2H)-

ylidene)methyl)-4,5-dihydrooxazol-5-yl)methoxy)carbonyl)-5-methylhex-2-enoic acid (24)

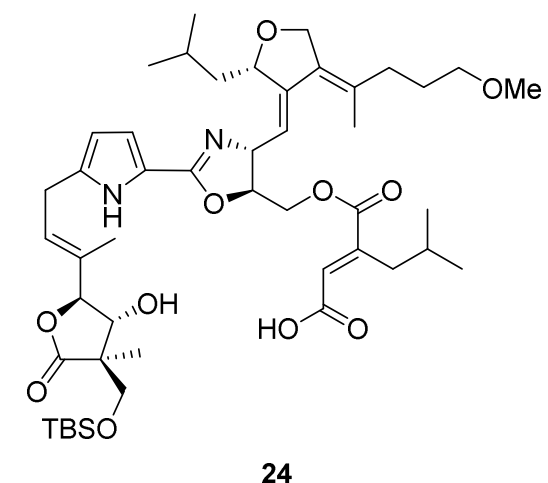

$\mathrm{C}_{47} \mathrm{H}_{72} \mathrm{~N}_{2} \mathrm{O}_{11} \mathrm{Si}, \mathrm{M}=869.2 \mathrm{~g} / \mathrm{mol}$

23a (3.7 mg, $4.06 \mu \mathrm{mol}, 1.0$ equiv) was dissolved in THF $(250 \mu \mathrm{L})$ at room temperature. Then $\mathrm{MeOH}(250 \mu \mathrm{L})$ was added followed by $1 \mathrm{M}$ aqueous $\mathrm{K}_{2} \mathrm{CO}_{3}$-solution $(250 \mu \mathrm{L})$. The resulting mixture was stirred at room temperature for 50 minutes before it was diluted with EtOAc $(2 \mathrm{~mL})$ and quenched by addition of a saturated solution of $\mathrm{NH}_{4} \mathrm{Cl}$ $(2 \mathrm{~mL})$. The layers were separated and the aqueous layer was extracted with EtOAc $(3 \times 2 \mathrm{~mL})$. The organic layers were combined, dried over $\mathrm{MgSO}_{4}$ and concentrated in vacuo. Purification by preparative TLC $\left(\mathrm{SiO}_{2}\right.$, cyclohexane/EtOAc/HOAc, 50:50:2) followed by co-evaporation with toluene to remove residual acetic acid gave $\mathbf{2 4}(3.10 \mathrm{mg}$, $3.57 \mu \mathrm{mol}, 88 \%$ ) as a colorless glas.

$\mathbf{R}_{\boldsymbol{f}}=0.37$ (cyclohexane/EtOAc/HOAc $\left.=50 / 50 / 2\right) ;[\alpha]_{D}^{20}=-53.4(c=0.6$ in $\mathrm{MeOH}) ;$

${ }^{1} \mathbf{H}-\mathbf{N M R}\left(700 \mathrm{MHz}, \mathrm{CD}_{3} \mathrm{OD}\right): \delta[\mathrm{ppm}] 0.10$ (s, 3H), 0.10 (s, 3H), 0.81 (dd, $J=6.2,3.9$ $\mathrm{Hz}, 6 \mathrm{H}), 0.90$ (s, 9H), $1.00(\mathrm{t}, J=6.5 \mathrm{~Hz}, 6 \mathrm{H}), 1.07$ (s, 3H), 1.37-1.43 (m, 1H), 1.59-1.65 $(\mathrm{m}, 1 \mathrm{H}), 1.68-1.76(\mathrm{~m}, 2 \mathrm{H}), 1.72-1.78(\mathrm{~m}, 1 \mathrm{H}), 1.78(\mathrm{~s}, 3 \mathrm{H}), 1.85-1.90(\mathrm{~m}, 1 \mathrm{H}), 1.98(\mathrm{~s}$, $3 \mathrm{H}), 2.07-2.17(\mathrm{~m}, 2 \mathrm{H}), 2.67(\mathrm{~d}, J=7.1 \mathrm{~Hz}, 2 \mathrm{H}), 3.35(\mathrm{~s}, 3 \mathrm{H}), 3.38(\mathrm{t}, J=6.0 \mathrm{~Hz}, 2 \mathrm{H})$, $3.51(\mathrm{~d}, J=6.0 \mathrm{~Hz}, 2 \mathrm{H}), 3.54(\mathrm{~d}, J=9.9 \mathrm{~Hz}, 1 \mathrm{H}), 3.83(\mathrm{~d}, J=9.9 \mathrm{~Hz}, 1 \mathrm{H}), 4.45-4.50$ (m, $1 \mathrm{H}), 4.47-4.52(\mathrm{~m}, 1 \mathrm{H}), 4.50-4.55(\mathrm{~m}, 1 \mathrm{H}), 4.53-4.57(\mathrm{~m}, 1 \mathrm{H}), 4.58-4.62(\mathrm{~m}, 1 \mathrm{H}), 4.61-$ $4.64(\mathrm{~m}, 1 \mathrm{H}), 4.65-4.70(\mathrm{~m}, 1 \mathrm{H}), 4.68-4.74(\mathrm{~m}, 1 \mathrm{H}), 4.97(\mathrm{~d}, J=10.8 \mathrm{~Hz}, 1 \mathrm{H}), 5.59(\mathrm{~d}, J$ $=9.9 \mathrm{~Hz}, 1 \mathrm{H}), 5.84(\mathrm{t}, J=6.8 \mathrm{~Hz}, 1 \mathrm{H}), 6.02(\mathrm{~d}, J=3.0 \mathrm{~Hz}, 1 \mathrm{H}), 6.77-6.79(\mathrm{~m}, 1 \mathrm{H}), 6.78-$ 
$6.79(\mathrm{~m}, 1 \mathrm{H}) ;{ }^{13} \mathbf{C}$ NMR $\left(175 \mathrm{MHz}, \mathrm{CD}_{3} \mathrm{OD}\right): \delta[\mathrm{ppm}]=-5.5,-5.5,11.5,13.3,19.0,20.3$, 22.1, 22.7, 22.7, 24.1, 26.1, 26.3 (3C), 27.1, 28.3, 29.6, 35.0, 36.9, 44.9, 51.4, 58.8, 65.5, $65.5,67.9,70.3,71.4,72.9,80.5,84.5,87.8,109.1,116.4,118.5,122.9,128.1,130.7$, $131.2,133.7,134.3,137.8,145.5,145.8,161.1,168.5,169.0,180.6$; HRMS (ESI+): calculated for $\mathrm{C}_{47} \mathrm{H}_{73} \mathrm{~N}_{2} \mathrm{O}_{11} \mathrm{Si}^{+}[\mathrm{M}+\mathrm{H}]^{+}:$: 869.4978, found: 869.4973.

$\left(1^{4} R, 1^{5} S, 6^{2} S, 6^{3} R, 6^{4} R, 4 E, 9 E\right)-6^{4}-(((t e r t-B u t y l d i m e t h y l s i l y l) o x y) m e t h y l)-10$-isobutyl-1 ${ }^{4}$ ((Z)-((S,Z)-2-isobutyl-4-(5-methoxypentan-2-ylidene)dihydrofuran-3(2H)ylidene)methyl)- $6^{4}, 5$-dimethyl- ${ }^{4}, 1^{5}, 6^{2}, 6^{3}, 6^{4}, 6^{5}$-hexahydro-2 ${ }^{1} H$-7,12-dioxa-1(2,5)oxazola-2(2,5)-pyrrola-6(2,3)-furanacyclotridecaphan-4,9-diene- $6^{5}, 8,11$-trione (25)

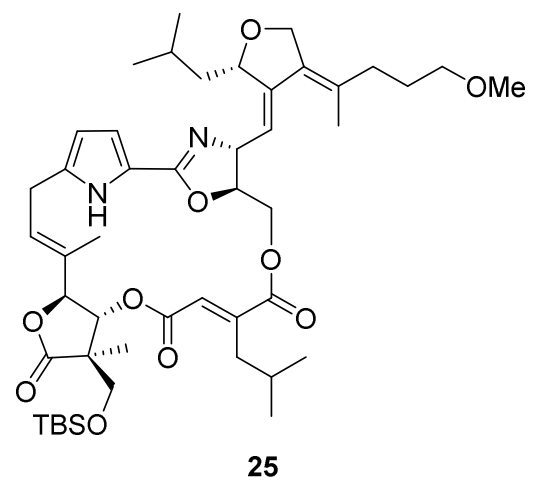

$\mathrm{C}_{47} \mathrm{H}_{70} \mathrm{~N}_{2} \mathrm{O}_{10} \mathrm{Si}, \mathrm{M}=851.2 \mathrm{~g} / \mathrm{mol}$

2-Methyl-6-nitrobenzoic anhydride (5.9 mg $17.3 \mu \mathrm{mol}$, 5.0 equiv), DMAP (3.4 mg, $27.6 \mu \mathrm{mol}, 8.0$ equiv) and molecular sieves (4 $\AA$ ) were dried under vacuum for 2 hours before $\mathrm{CH}_{2} \mathrm{Cl}_{2}(2.0 \mathrm{~mL})$ was added. To this, a solution of $23(3.0 \mathrm{mg}, 3.45 \mu \mathrm{mol}$, 1.0 equiv) in $\mathrm{CH}_{2} \mathrm{Cl}_{2}(1.5 \mathrm{~mL})$ was added over a period of 16 hours by a syringe pump. After 4 more hours at room temperature, the reaction mixture was filtered and an aqueous solution of $\mathrm{NaHCO}_{3}(2 \mathrm{~mL})$ was added. The layers were separated and the aqueous layer was extracted with EtOAc $(3 \times 3 \mathrm{~mL})$. The organic layers were combined, dried over $\mathrm{MgSO}_{4}$ and concentrated in vacuo. Purification by preparative TLC $\left(\mathrm{SiO}_{2}\right.$, cyclohexane/EtOAc, 1:1) gave 25 (2.7 mg, $3.17 \mu \mathrm{mol}, 92 \%)$ as a colorless gum.

$\mathbf{R}_{\boldsymbol{f}}=0.48$ (cyclohexane/EtOAc $\left.=2 / 1\right) ;[\alpha]_{D}^{20}=-101.2\left(c=0.5\right.$ in $\left.\mathrm{CH}_{2} \mathrm{Cl}_{2}\right) ;{ }^{1} \mathbf{H}-\mathbf{N M R}$ $\left(700 \mathrm{MHz}, \mathrm{CD}_{2} \mathrm{Cl}_{2}\right): \delta[\mathrm{ppm}]=0.09(\mathrm{~s}, 3 \mathrm{H}), 0.10(\mathrm{~s}, 3 \mathrm{H}), 0.78(\mathrm{~d}, J=6.7 \mathrm{~Hz}, 3 \mathrm{H}), 0.82$ (d, $J=6.5 \mathrm{~Hz}, 3 \mathrm{H}), 0.91$ (s, 9H), 0.96 (d, $J=6.2 \mathrm{~Hz}, 6 \mathrm{H}), 1.06$ (s, 3H), 1.37-1.43 (m, 
1H), 1.53-1.58 (m, 1H), 1.62-1.73 (m, 2H), 1.69-1.76 (m, 1H), 1.81 (s, 3H), 1.81-1.87 (m, $1 \mathrm{H}), 1.87(\mathrm{~s}, 3 \mathrm{H}), 2.00-2.08(\mathrm{~m}, 2 \mathrm{H}), 2.38-2.52(\mathrm{~m}, 2 \mathrm{H}), 3.25-3.31(\mathrm{~m}, 1 \mathrm{H}), 3.28(\mathrm{~s}, 3 \mathrm{H})$, $3.31(\mathrm{t}, J=6.2 \mathrm{~Hz}, 2 \mathrm{H}), 3.55(\mathrm{dd}, J=14.5,11.5 \mathrm{~Hz}, 1 \mathrm{H}), 3.70(\mathrm{~d}, J=9.5 \mathrm{~Hz}, 1 \mathrm{H}), 3.87$ $(\mathrm{d}, J=9.5 \mathrm{~Hz}, 1 \mathrm{H}), 4.32(\mathrm{~d}, J=12.3 \mathrm{~Hz}, 1 \mathrm{H}), 4.42(\mathrm{~d}, J=12.0 \mathrm{~Hz}, 1 \mathrm{H}), 4.47(\mathrm{~d}, J=9.7$ $\mathrm{Hz}, 1 \mathrm{H}), 4.49$ (d, $J=13.6 \mathrm{~Hz}, 1 \mathrm{H}), 4.56(\mathrm{dd}, J=9.8,2.0 \mathrm{~Hz}, 1 \mathrm{H}), 4.59$ (d, $J=12.3 \mathrm{~Hz}$, 1H), 4.64 (br. s., $1 \mathrm{H}), 4.86$ (d, $J=10.5 \mathrm{~Hz}, 1 \mathrm{H}), 5.43$ (d, $J=12.3 \mathrm{~Hz}, 1 \mathrm{H}), 5.46$ (d, $J=$ $10.3 \mathrm{~Hz}, 1 \mathrm{H}), 5.79$ (d, $J=9.3 \mathrm{~Hz}, 1 \mathrm{H}), 6.01$ (br. s., 1H), 6.60 (br. s., 1H), 6.66 (s, 1H), 9.98 (br. s., $1 \mathrm{H}) ;{ }^{13} \mathrm{C}-\mathrm{NMR}\left(175 \mathrm{MHz}, \mathrm{CD}_{2} \mathrm{Cl}_{2}\right): \delta[\mathrm{ppm}]=-5.4,-5.4,10.5,13.7,18.7$, 20.4, 21.9, 22.7, 22.7, 24.0, 25.6, 26.1 (3C), 27.4, 27.9, 29.0, 34.6, 37.1, 43.7, 50.0, 58.8, $65.6,67.0,67.3,69.8,72.4,73.1,79.9,83.6,84.5,108.8,114.6,119.4,121.4,128.5$, $129.9,130.6,131.9,133.5,136.6,144.6,145.2,159.9,165.9,166.7,176.8$; HRMS (ESI+): calculated for $\mathrm{C}_{47} \mathrm{H}_{71} \mathrm{~N}_{2} \mathrm{O}_{10} \mathrm{Si}^{+}[\mathrm{M}+\mathrm{H}]^{+}:$: 851.4872, found: 851.4870.

\section{Leupyrrin $B_{1}$ (2)}

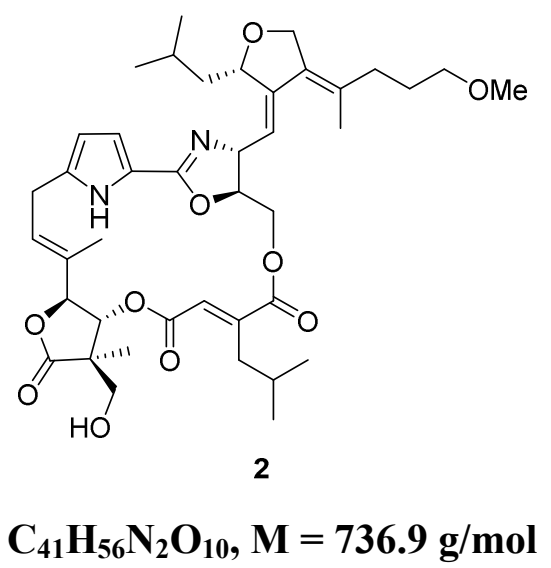

To a solution of $25\left(2.5 \mathrm{mg}, 2.94 \mu \mathrm{mol}, 1.0\right.$ equiv) in $\mathrm{MeCN}(50 \mu \mathrm{L})$ at $0{ }^{\circ} \mathrm{C}$ a solution of TASF ( $90 \%, 1.8 \mathrm{mg}, 5.88 \mu \mathrm{mol}, 2.0$ equiv) in $\mathrm{MeCN}(5 \mu \mathrm{L})$ was added. The resulting mixture allowed to warm up to room temperature. After 30 minutes the solution was cooled to $0{ }^{\circ} \mathrm{C}$ before further TASF $(90 \%, 1.8 \mathrm{mg}, 5.88 \mu \mathrm{mol}, 2.0$ equiv) in MeCN (5 $\mu \mathrm{L})$ was added. The resulting mixture allowed to warm up to room temperature. After 30 minutes the solution was cooled to $0{ }^{\circ} \mathrm{C}$ again before further TASF $(90 \%, 1.8 \mathrm{mg}$, $5.88 \mu \mathrm{mol}, 2.0$ equiv) in $\mathrm{MeCN}(5 \mu \mathrm{L})$ was added. The ice bath was removed and the stirring was continued for 30 minutes at room temperature. The reaction mixture was diluted with EtOAc $(2 \mathrm{~mL})$ and quenched by addition of a saturated solution of $\mathrm{NH}_{4} \mathrm{Cl}$ 
$(2 \mathrm{~mL})$. The layers were separated and the aqueous layer was extracted with EtOAc $(3 \times 2 \mathrm{~mL})$. The organic layers were combined, dried over $\mathrm{MgSO}_{4}$ and concentrated in vacuo. Purification by preparative TLC $\left(\mathrm{SiO}_{2}\right.$, cyclohexane/EtOAc, 50:50) gave leupyrrin $\mathrm{B}_{1}$ (2) $(0.9 \mathrm{mg}, 1.22 \mu \mathrm{mol}, 42 \%$, (74 \% brsm)).

$\mathbf{R}_{\boldsymbol{f}}=0.24$ (cyclohexane/EtOAc $\left.=2 / 1\right) ; \quad[\alpha]_{D}^{20}=+3.0 \quad(c=0.1$ in $\mathrm{MeOH}) ;{ }^{1} \mathbf{H}-\mathbf{N M R}$ (700 MHz, CD $\mathrm{CDD}_{3} \mathrm{OD} \delta=0.82(\mathrm{~s}, 3 \mathrm{H}), 0.83$ (s, 3H), 0.97 (d, $\left.J=6.7 \mathrm{~Hz}, 3 \mathrm{H}\right), 1.01$ (d, $J=6.5 \mathrm{~Hz}, 3 \mathrm{H}), 1.03$ (s, 3H), 1.37 (ddd, $J=13.9,9.7,2.0 \mathrm{~Hz}, 1 \mathrm{H}), 1.58$ (ddd, $J=14.3$, 10.8, 4.0 Hz, 1H), 1.63-1.78 (m, 3H), 1.75 (s, 3H), 1.83-1.89 (m, 1H), 1.92 (s, 3H), 2.01$2.14(\mathrm{~m}, 2 \mathrm{H}), 2.51$ (dd, $J=12.6,7.3 \mathrm{~Hz}, 1 \mathrm{H}), 2.57$ (dd, $J=12.6,7.3 \mathrm{~Hz}, 1 \mathrm{H}), 3.24$ (dd, $J=14.2,4.9 \mathrm{~Hz}, 1 \mathrm{H}), 3.31(\mathrm{~s}, 3 \mathrm{H}), 3.35$ (t, $J=6.1 \mathrm{~Hz}, 2 \mathrm{H}), 3.59$ (dd, $J=14.3,10.9 \mathrm{~Hz}$, 1H), $3.70(\mathrm{~d}, J=10.5 \mathrm{~Hz}, 1 \mathrm{H}), 3.78(\mathrm{~d}, J=10.8 \mathrm{~Hz}, 1 \mathrm{H}), 4.45$ (d, $J=12.3 \mathrm{~Hz}, 1 \mathrm{H}), 4.51$ $(\mathrm{dd}, J=12.7,2.4 \mathrm{~Hz}, 1 \mathrm{H}), 4.53-4.57(\mathrm{~m}, 1 \mathrm{H}), 4.61(\mathrm{dd}, J=10.1,4.1 \mathrm{~Hz}, 1 \mathrm{H}), 4.66(\mathrm{~d}$, $J=12.3 \mathrm{~Hz}, 1 \mathrm{H}), 4.68(\mathrm{~d}, J=9.3 \mathrm{~Hz}, 1 \mathrm{H}), 4.67-4.70(\mathrm{~m}, 1 \mathrm{H}), 4.88-4.90(\mathrm{~m}, 1 \mathrm{H}), 4.98$ (dt, $J=10.8,1.9 \mathrm{~Hz}, 1 \mathrm{H}), 5.42$ (d, $J=9.0 \mathrm{~Hz}, 1 \mathrm{H}), 5.49$ (d, $J=9.9 \mathrm{~Hz}, 1 \mathrm{H}), 5.83$ (dd, $J=10.2,4.6 \mathrm{~Hz}, 1 \mathrm{H}), 5.97(\mathrm{~d}, J=3.4 \mathrm{~Hz}, 1 \mathrm{H}), 6.58(\mathrm{~s}, 1 \mathrm{H}), 6.70(\mathrm{~d}, J=3.7 \mathrm{~Hz}, 1 \mathrm{H})$ ppm; ${ }^{13} \mathbf{C}$ NMR (175 MHz, $\left.\mathrm{CD}_{3} \mathrm{OD}\right): \delta 9.9,13.6,20.2,22.0,22.8,22.8,24.1,26.1,27.1$, 28.3, 29.6, 34.9, 37.1, 44.6, 50.9, 58.8, 65.2, 66.8, 68.8, 70.3, 72.8, 74.6, 80.6, 84.1, 85.1, 109.1, 115.6, 119.4, 123.3, 127.9, 130.9, 131.2, 131.5, 134.0, 136.8, 144.5, 148.1, 161.3, 166.1, 167.5, 178.9; HRMS (ESI+): calculated for $\mathrm{C}_{41} \mathrm{H}_{57} \mathrm{~N}_{2} \mathrm{O}_{10}{ }^{+}[\mathrm{M}+\mathrm{H}]^{+}: 737.4008$, found: 737.3993 . 
3 Copies of NMR spectra

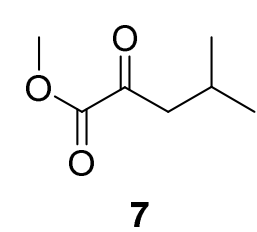

1H-NMR (300 MHz)

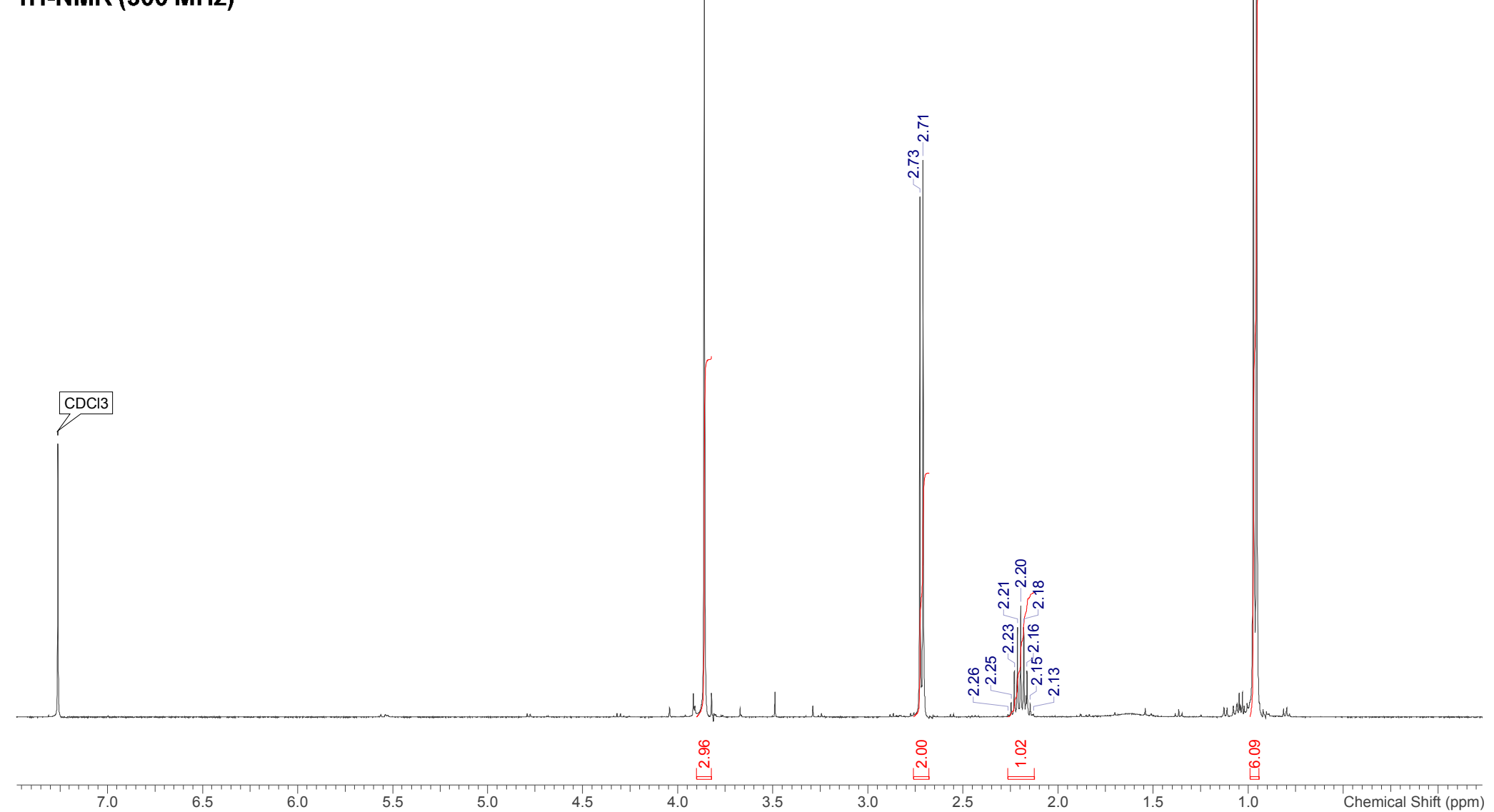



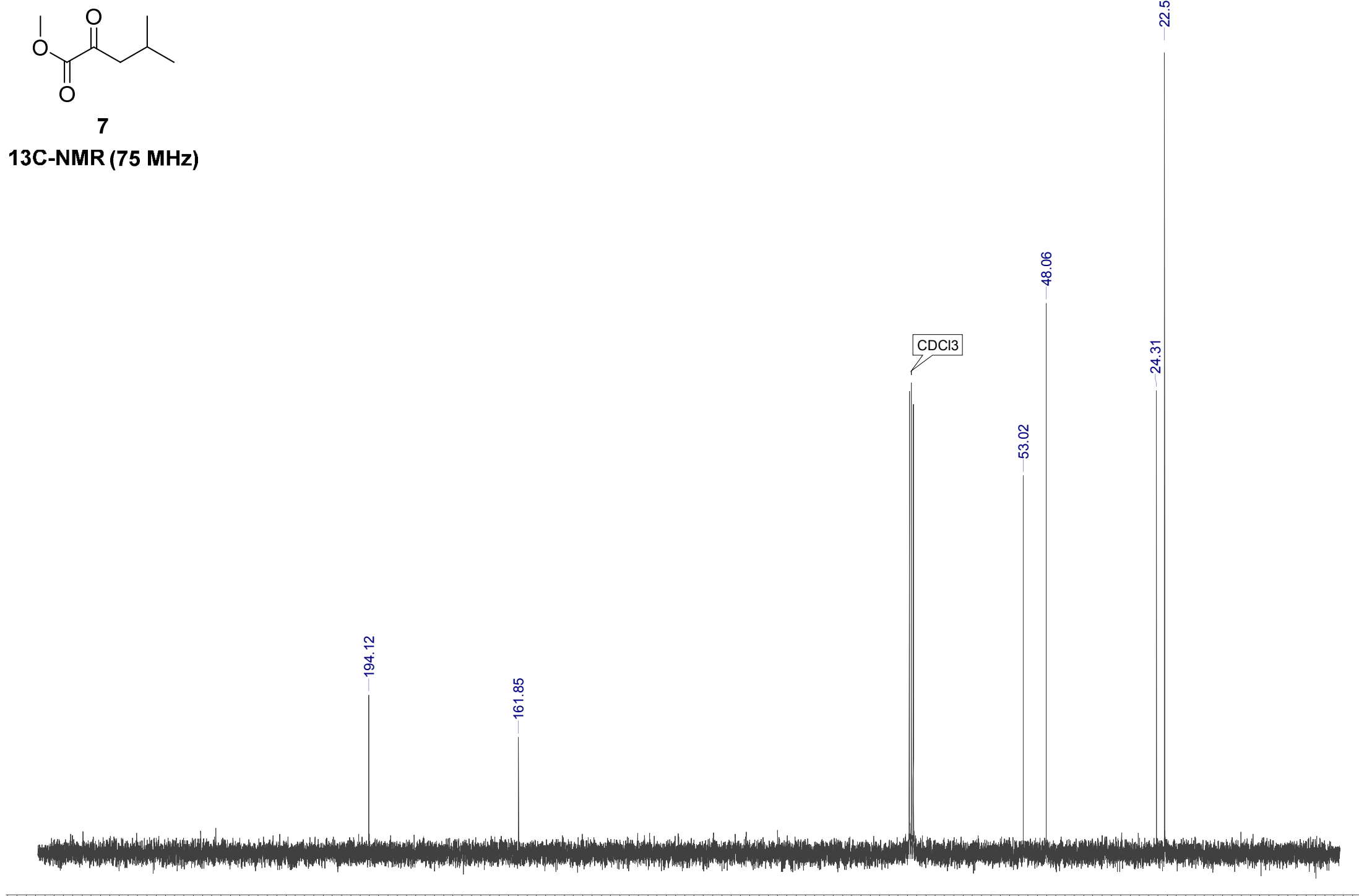


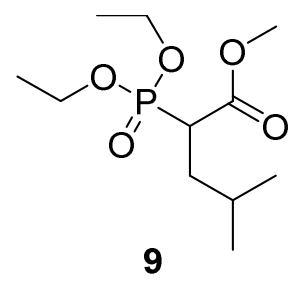

1H-NMR (400 MHz)

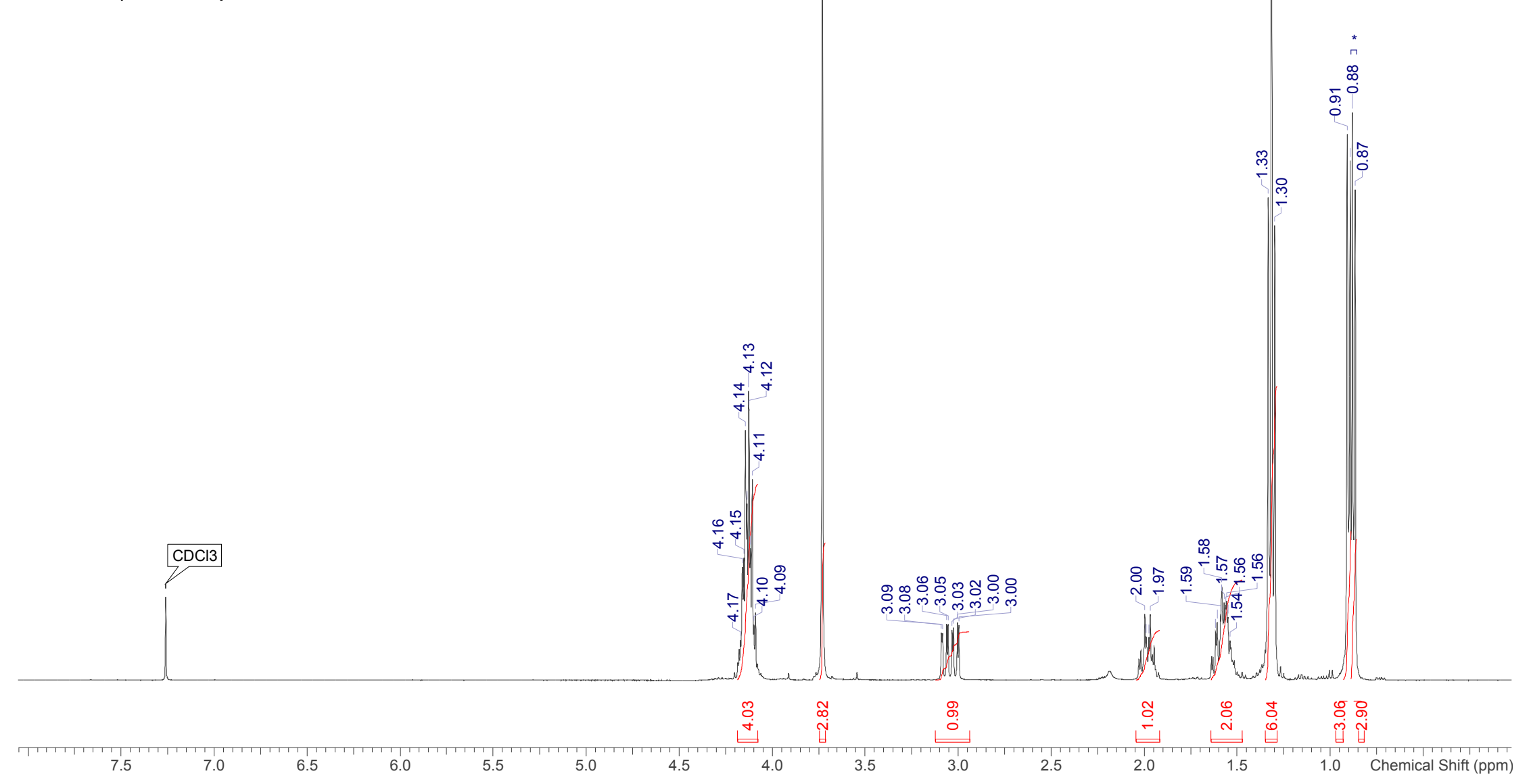




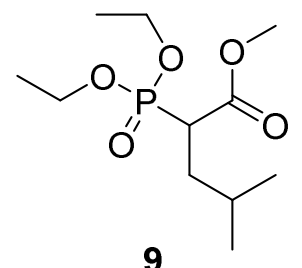

13C-NMR (100 MHz)
$\mathrm{CDCl3}$

กี

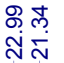

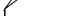

ֻู

กู่
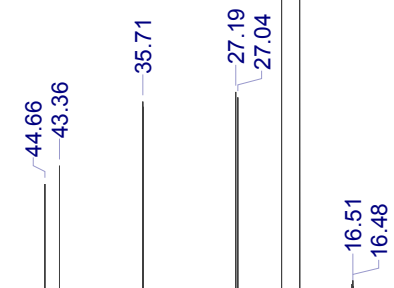

$\stackrel{\infty}{\stackrel{0}{8}}$

WM. 


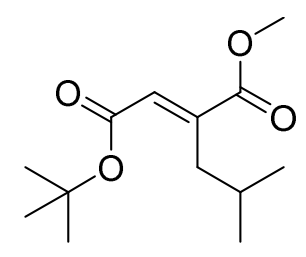

12

1H-NMR (500 MHz)

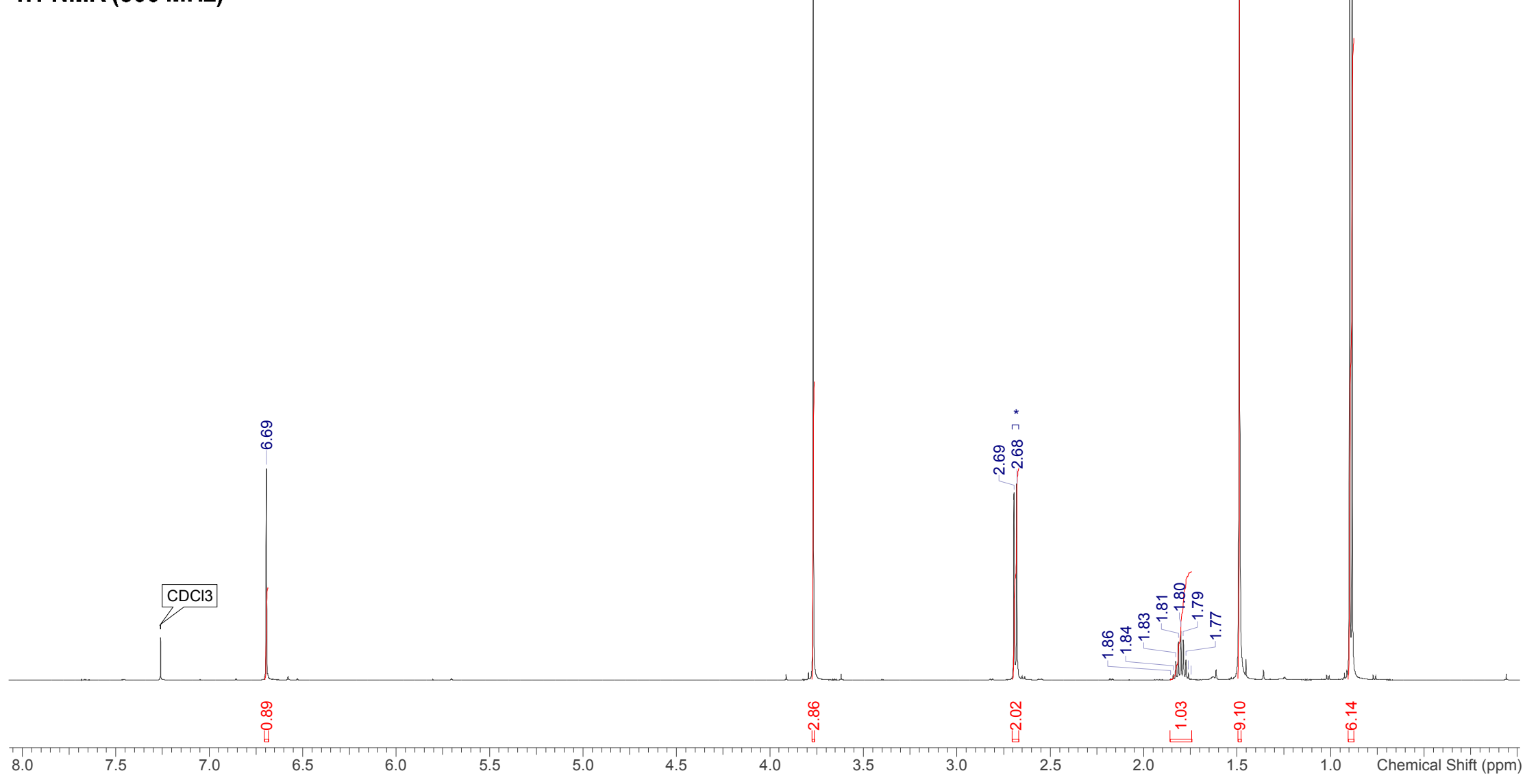




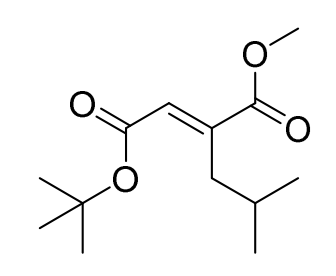

12

13C-NMR (75 MHz)

\section{C-NMR (75 MHz)}

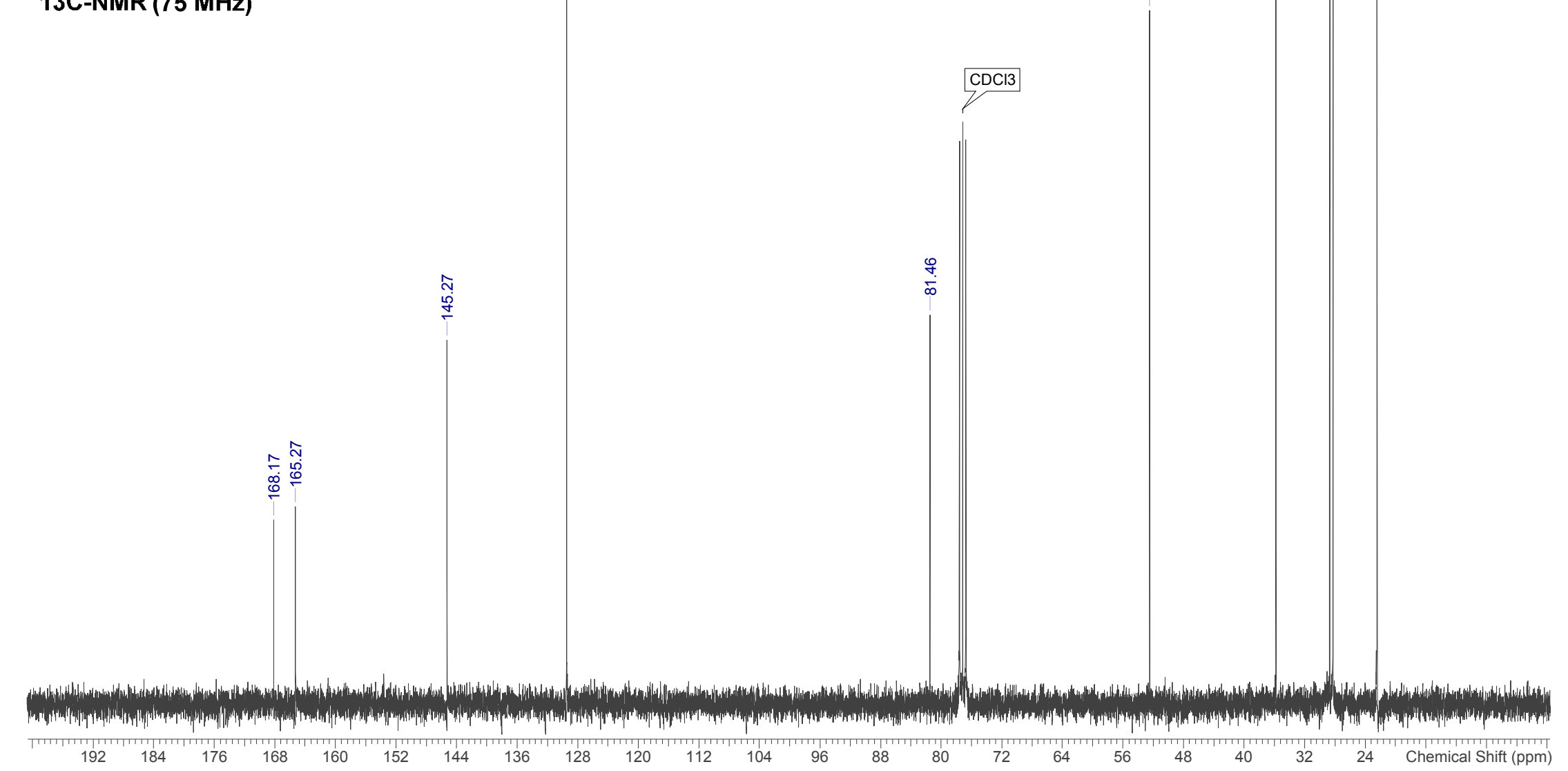




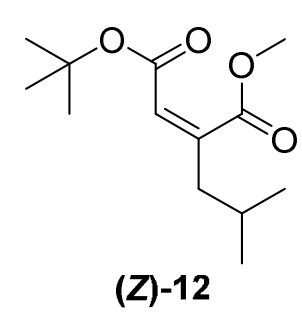

1H-NMR (300 MHz)

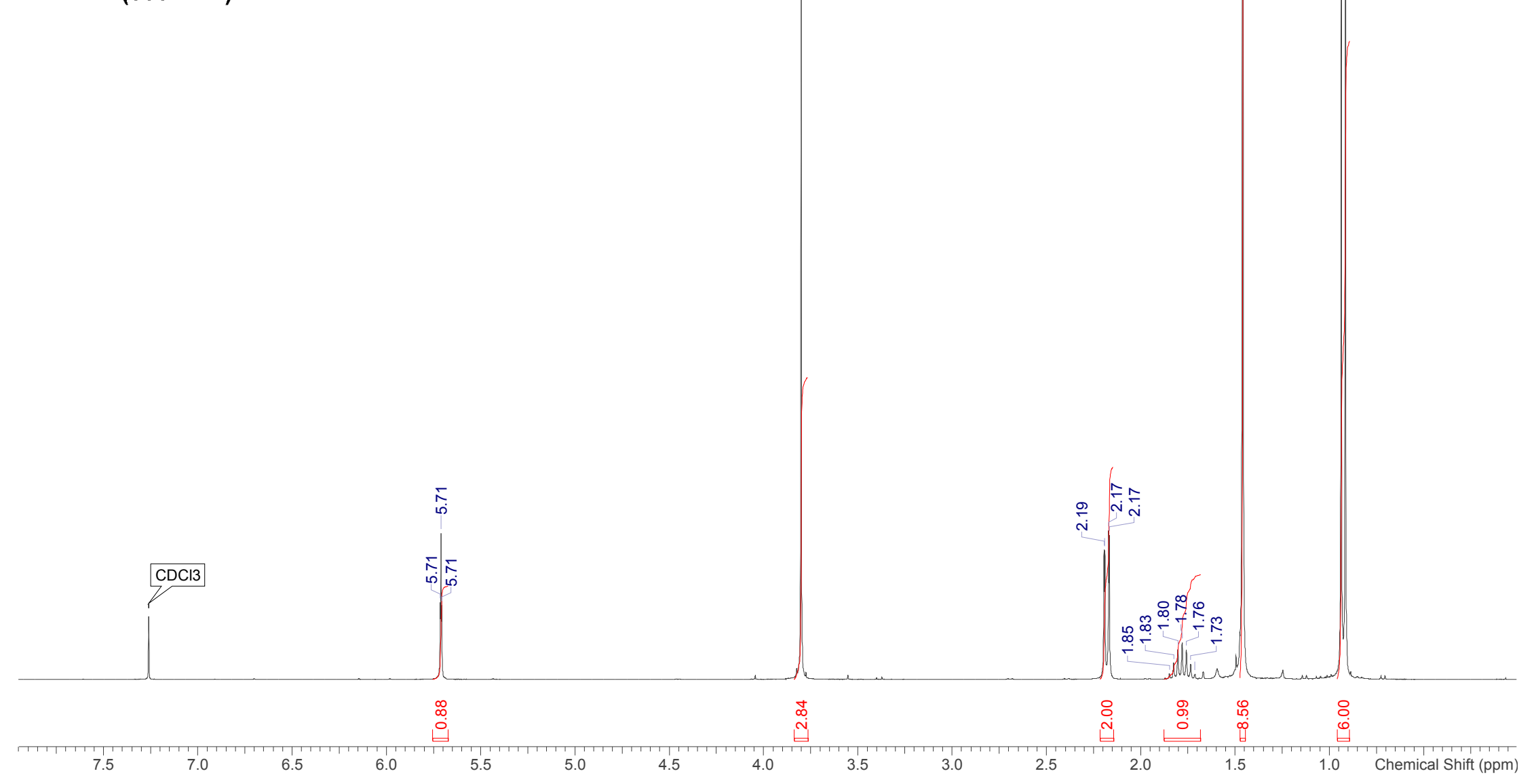




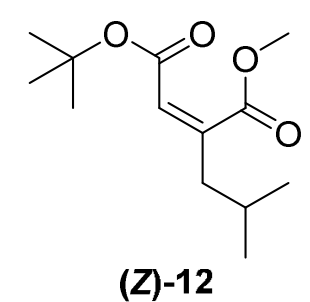

13C-NMR (75 MHz)

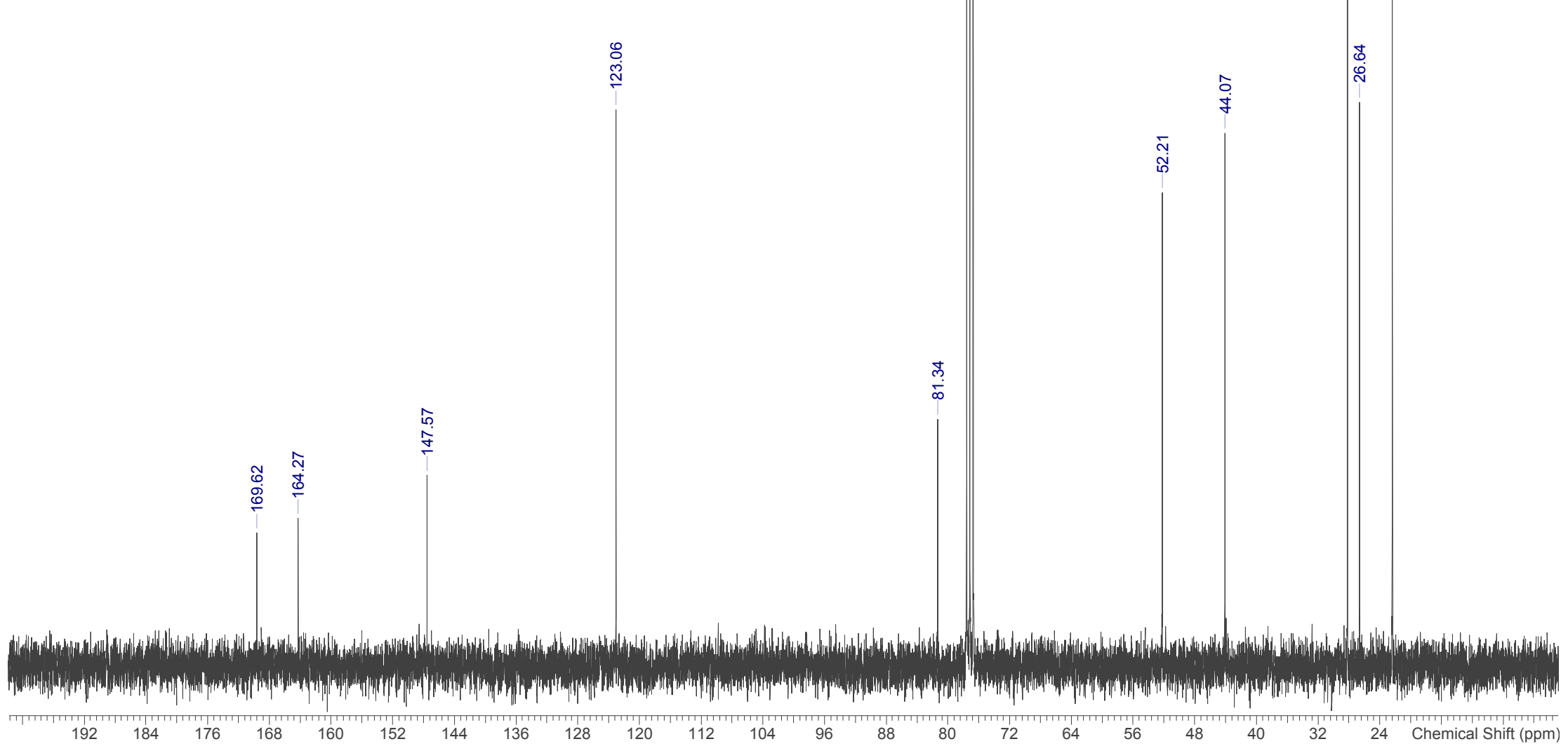




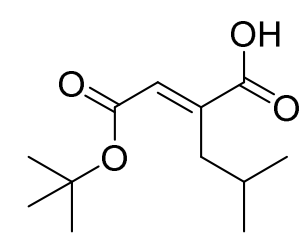

14

1H-NMR (400 MHz)

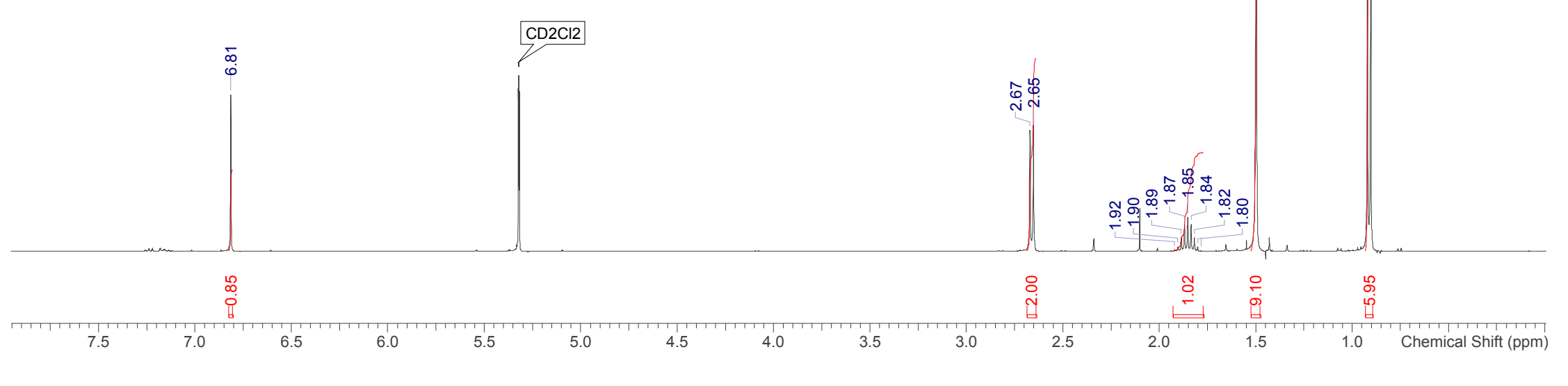




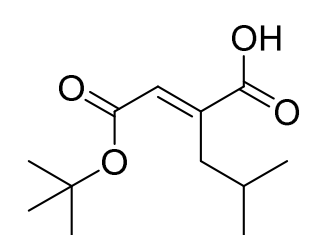

14

13C-NMR (100 MHz)

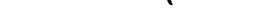




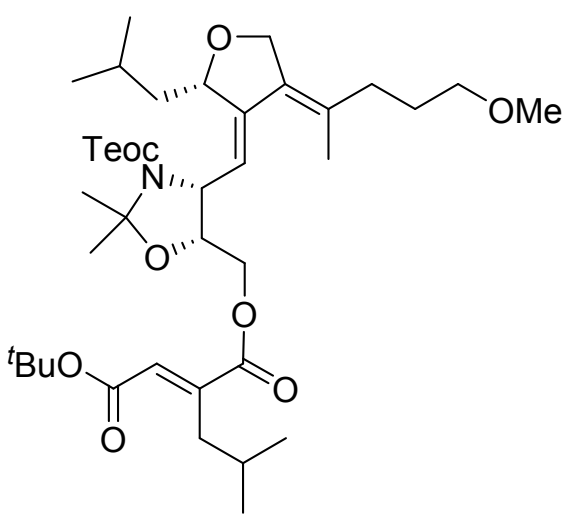

15

1H NMR (700 MHz, 339 K)

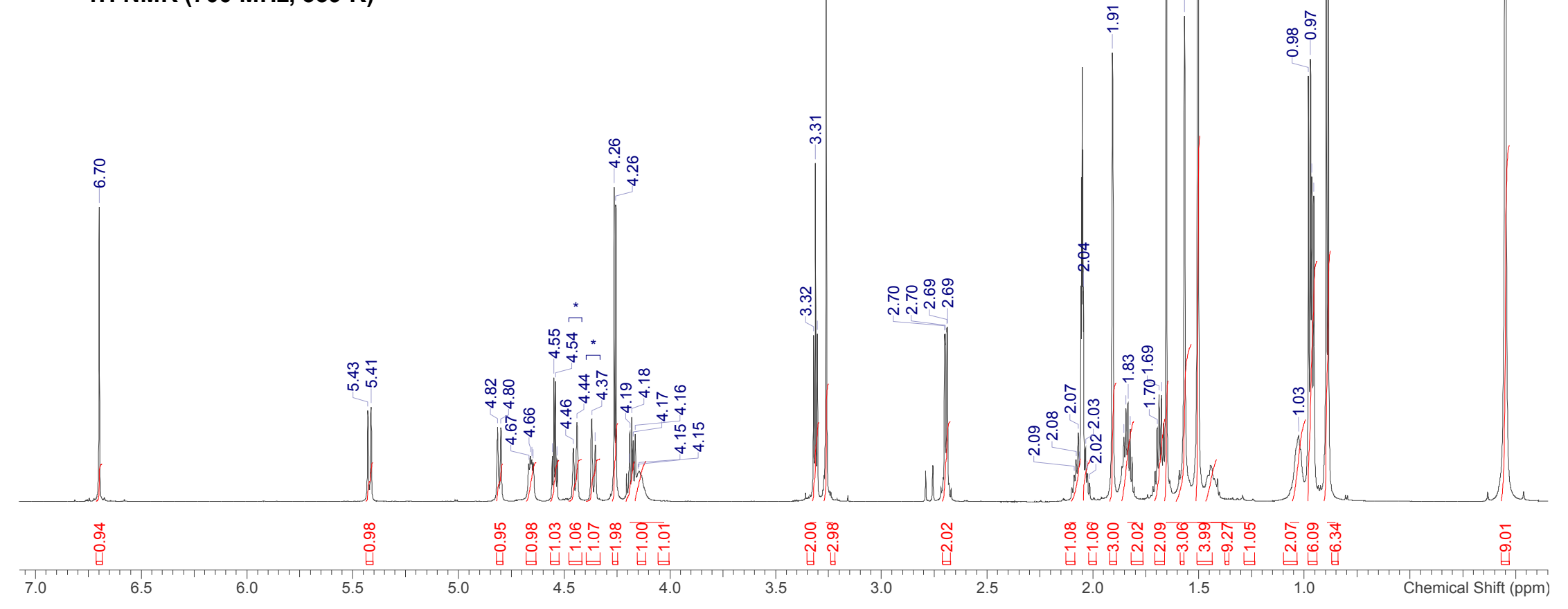




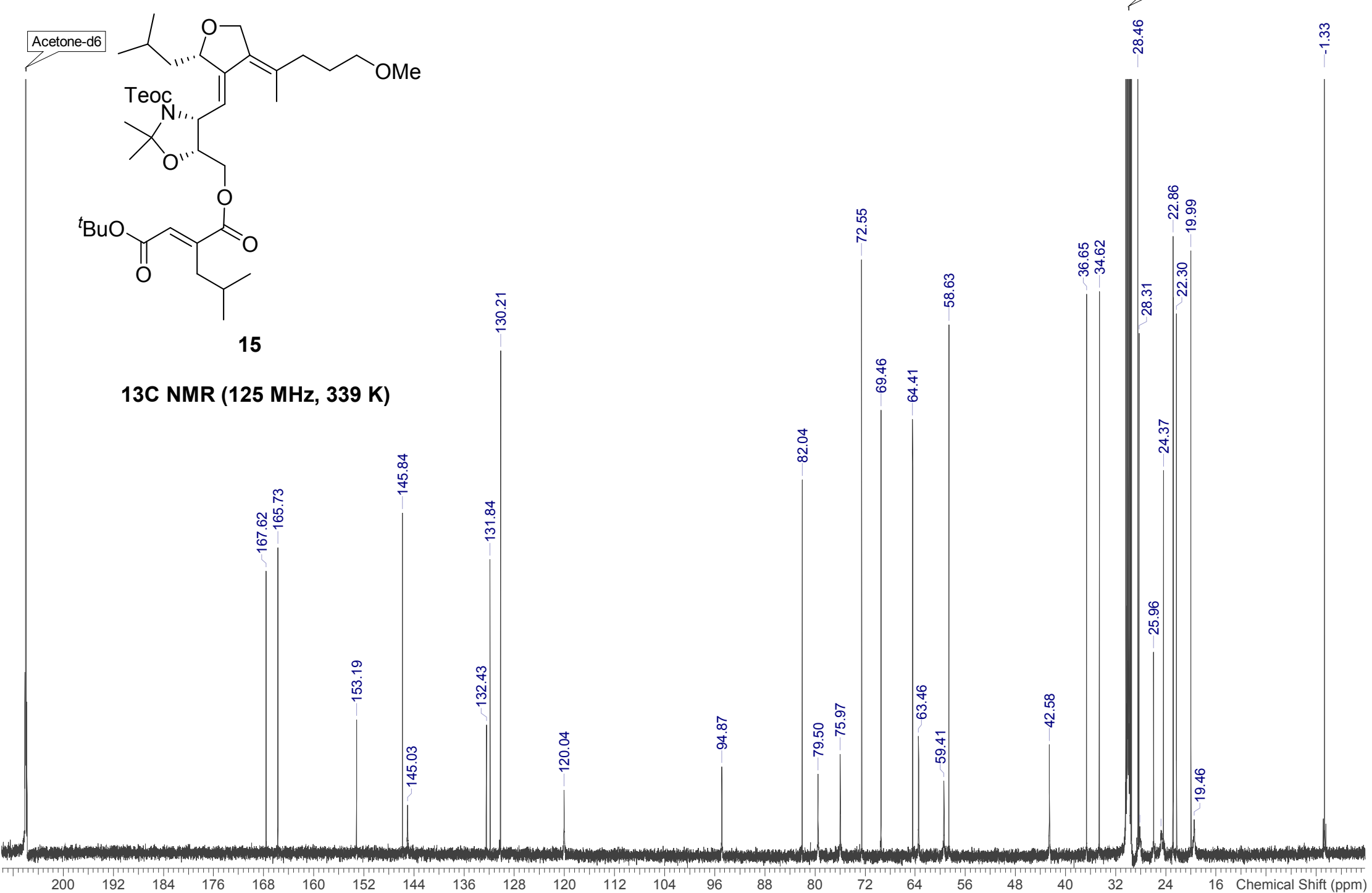




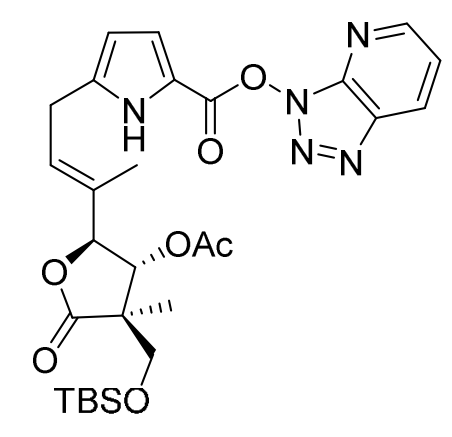

22

1H NMR (400 MHz)

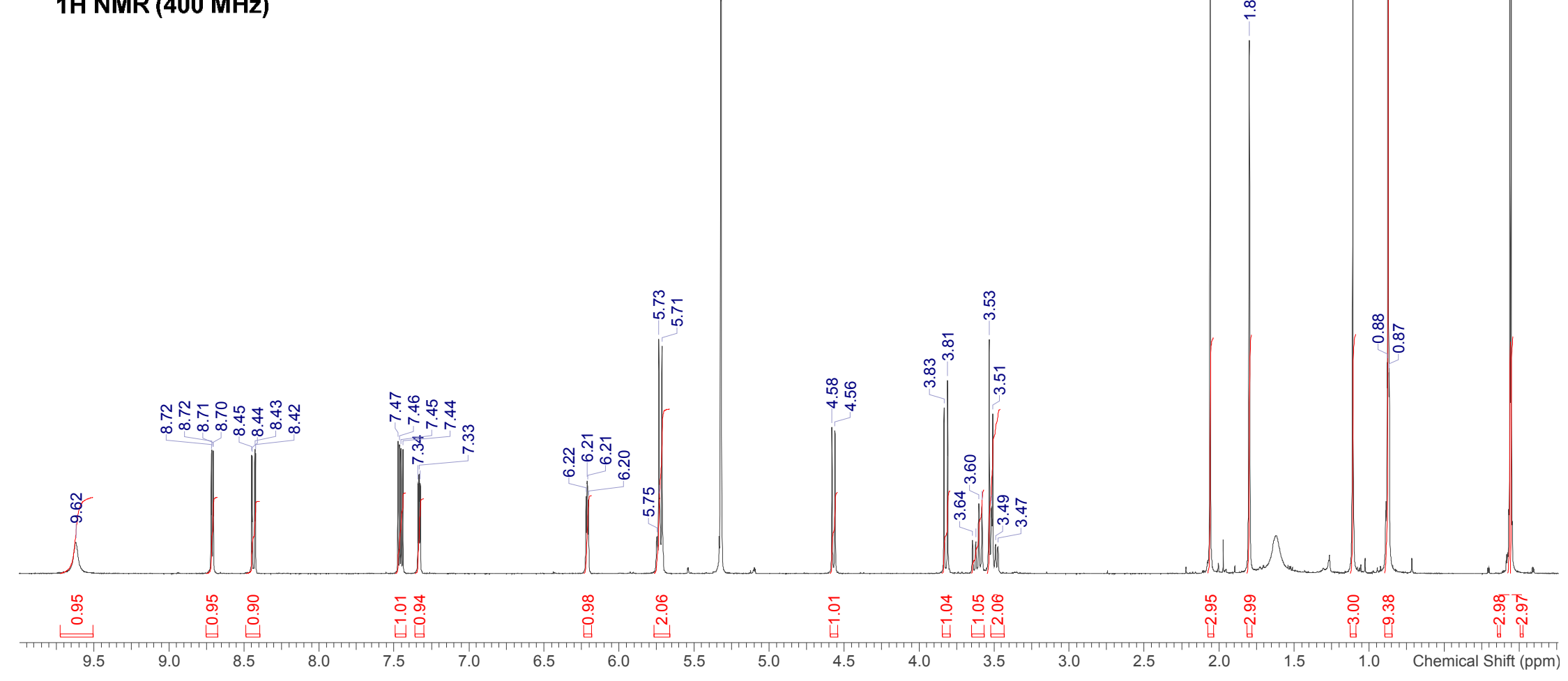




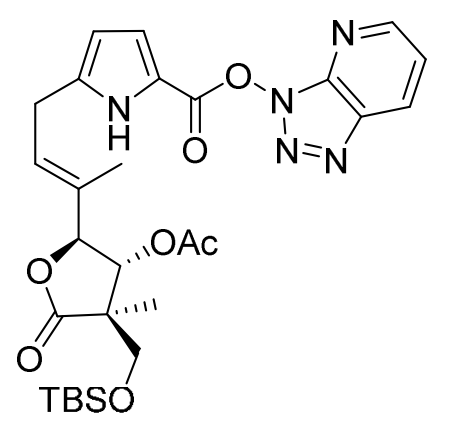

22

13C NMR (125 MHz)

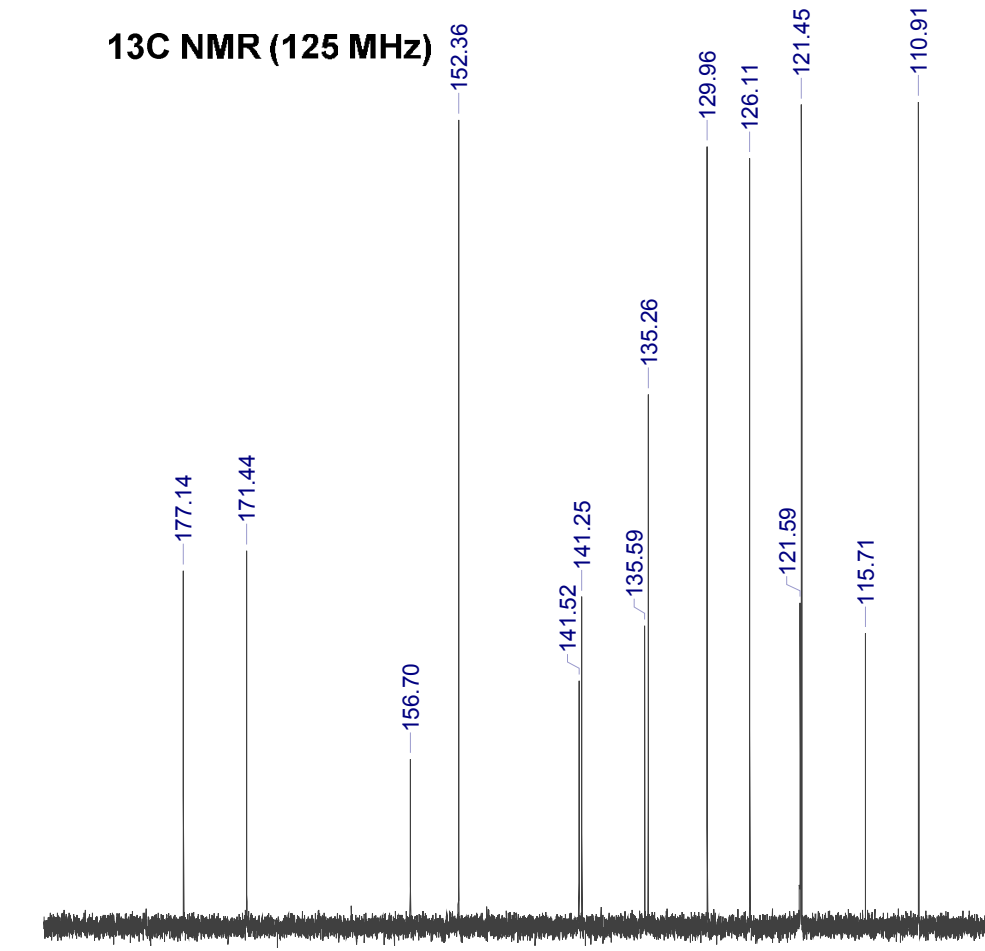

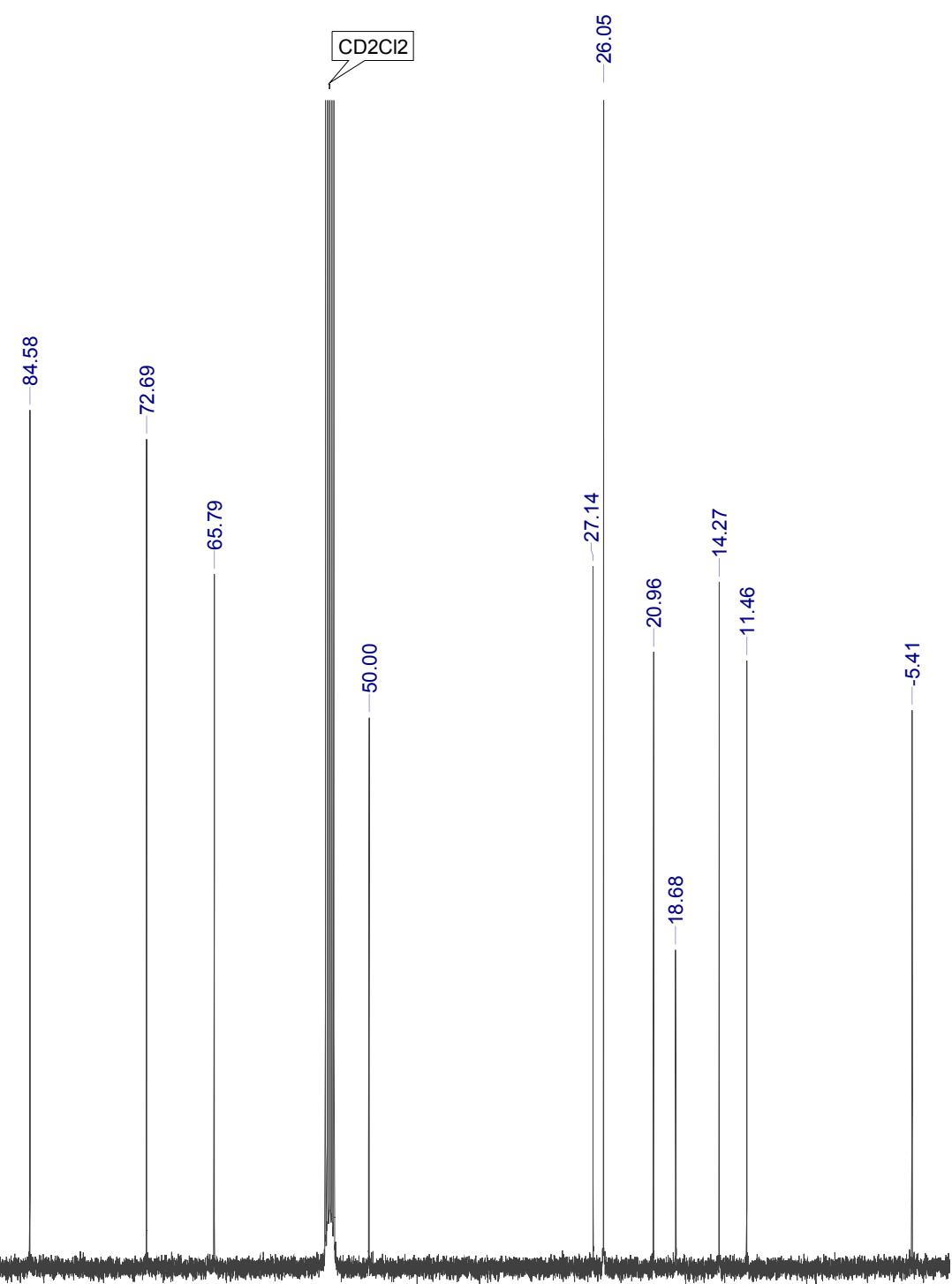

88 


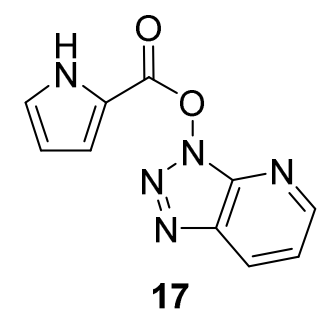

1H NMR (300 MHz)

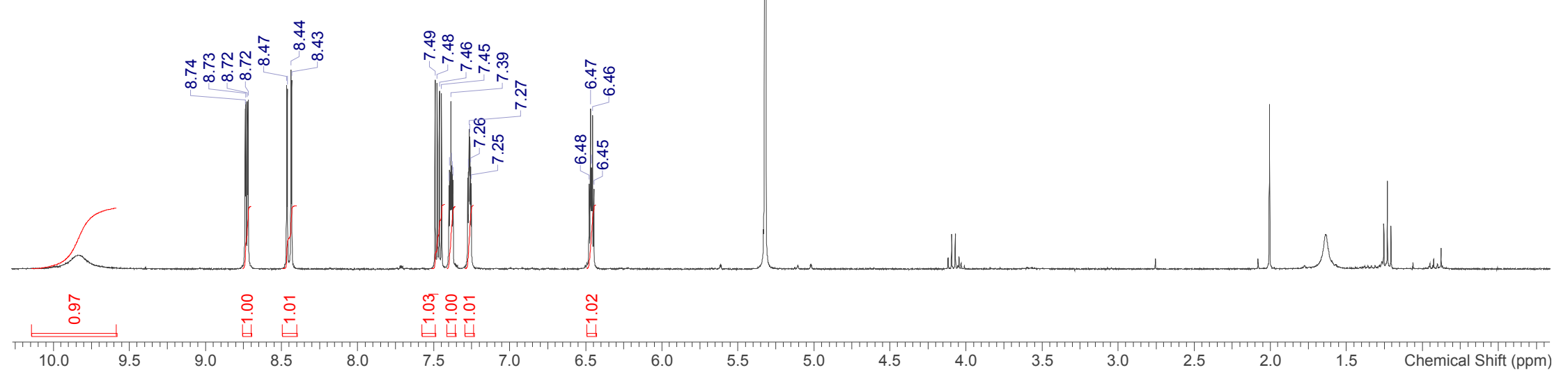



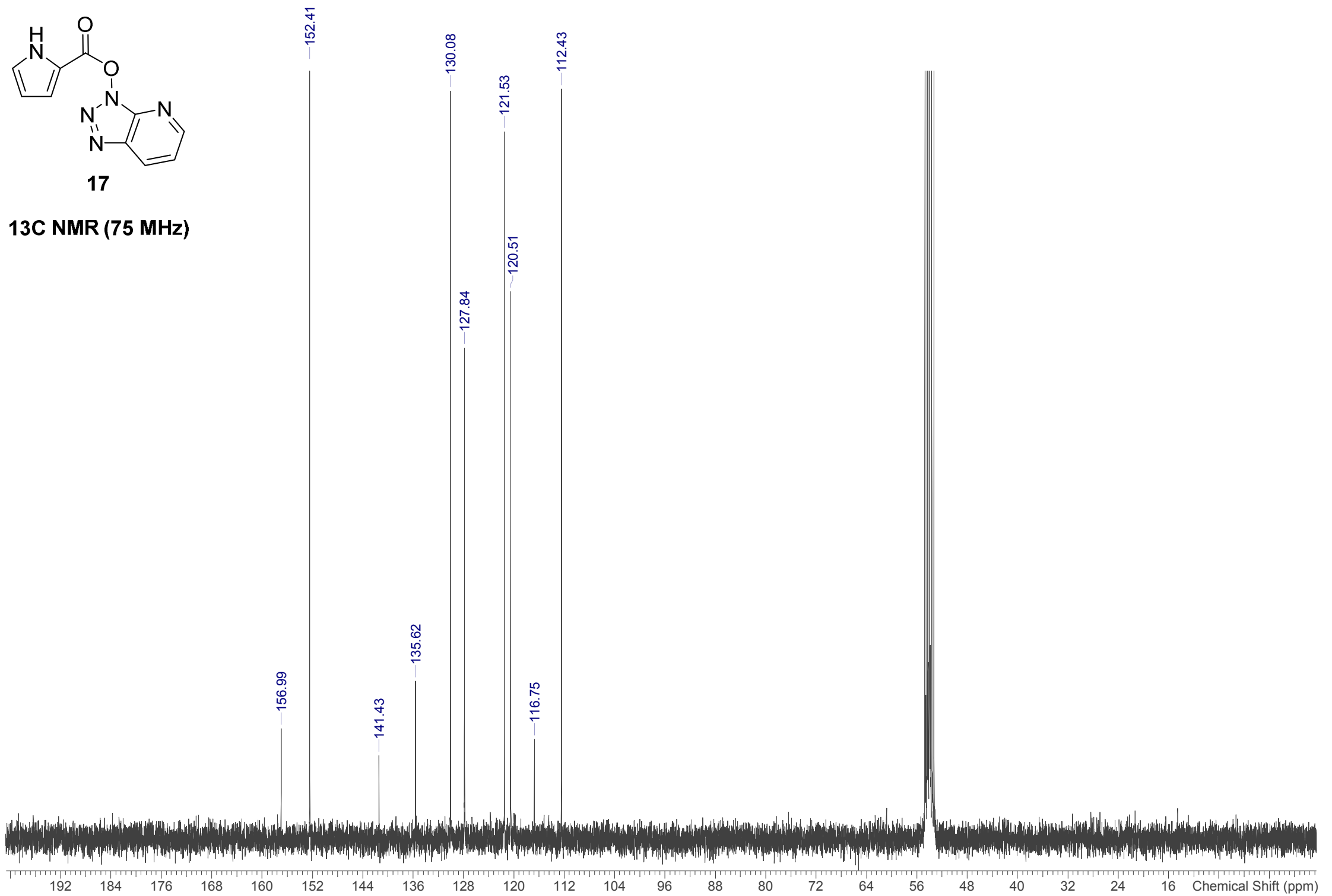


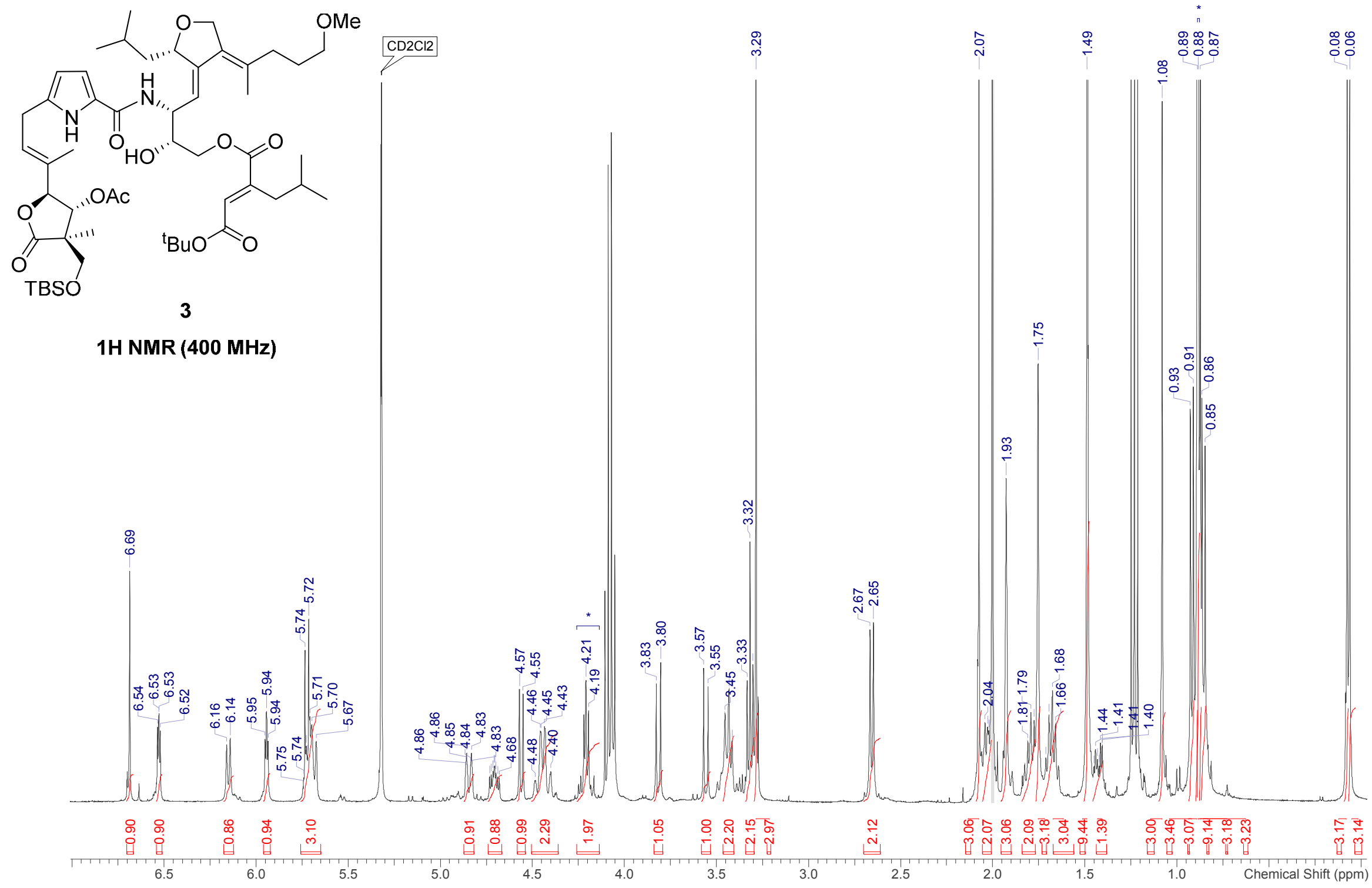



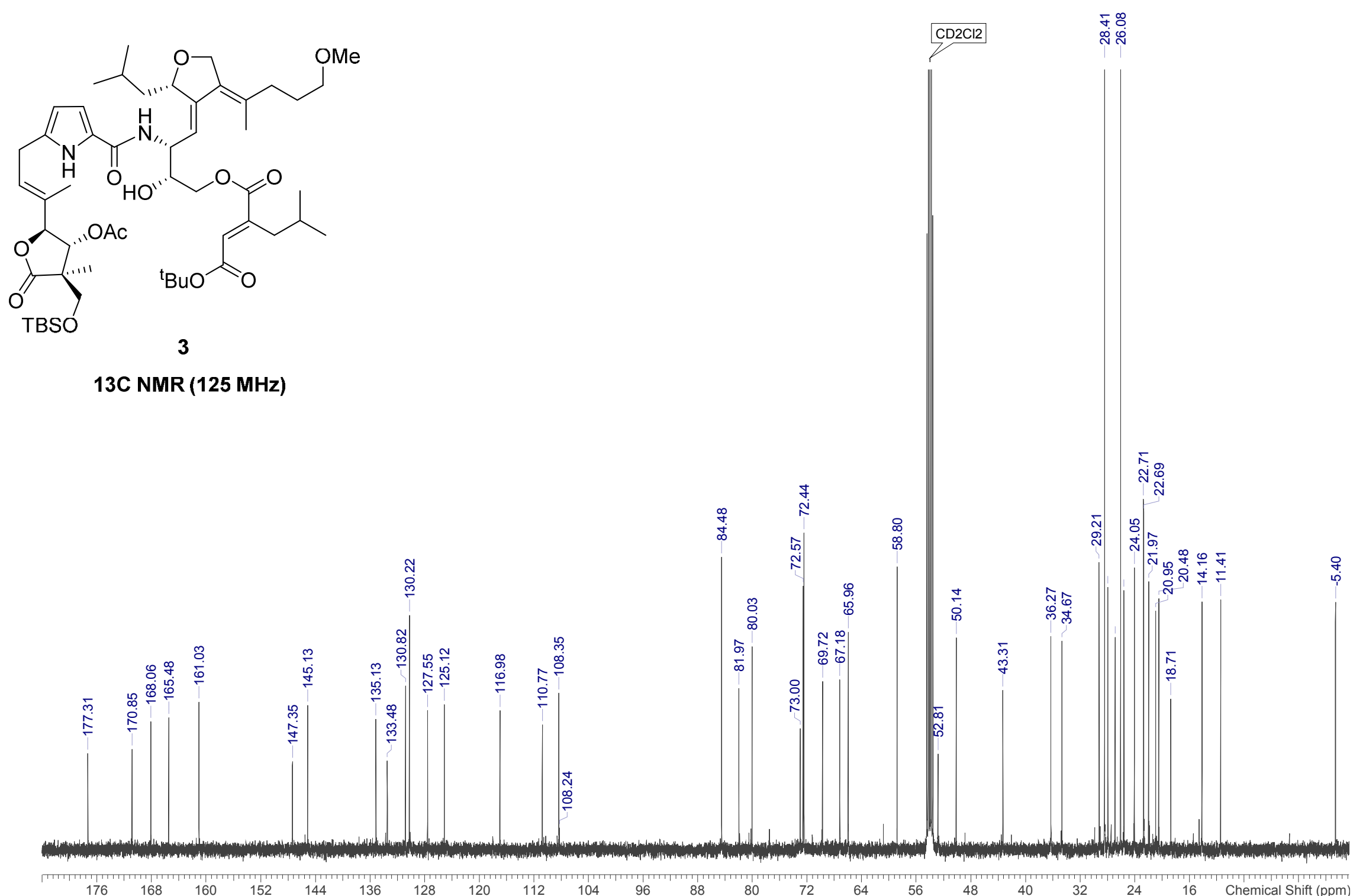


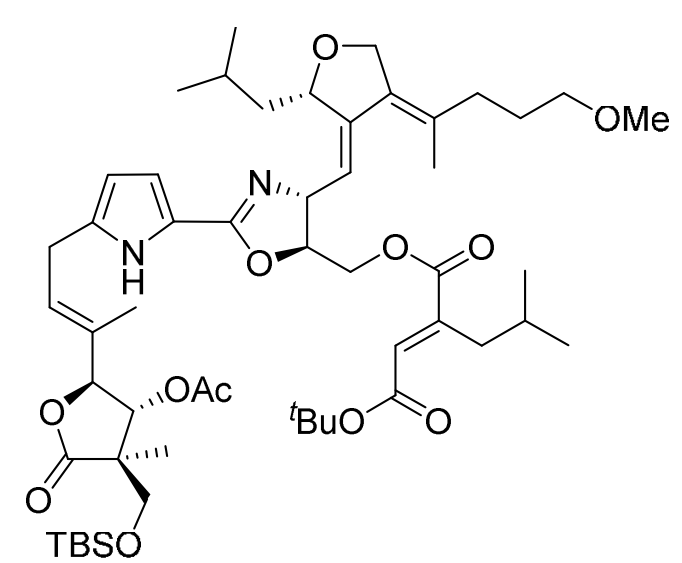

23

1H NMR (400 MHz)

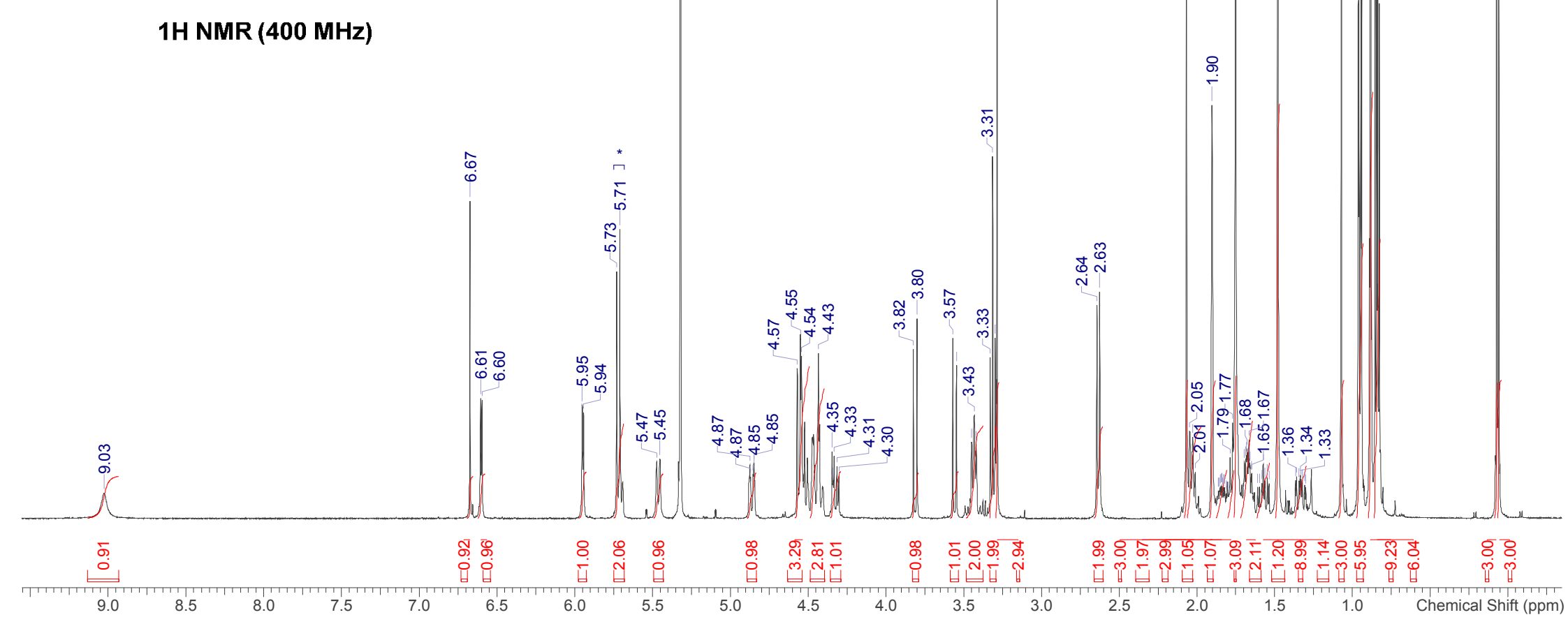




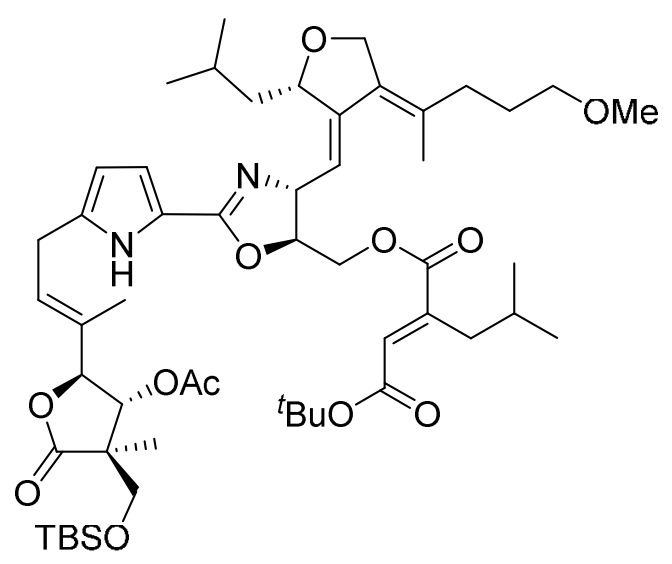

23

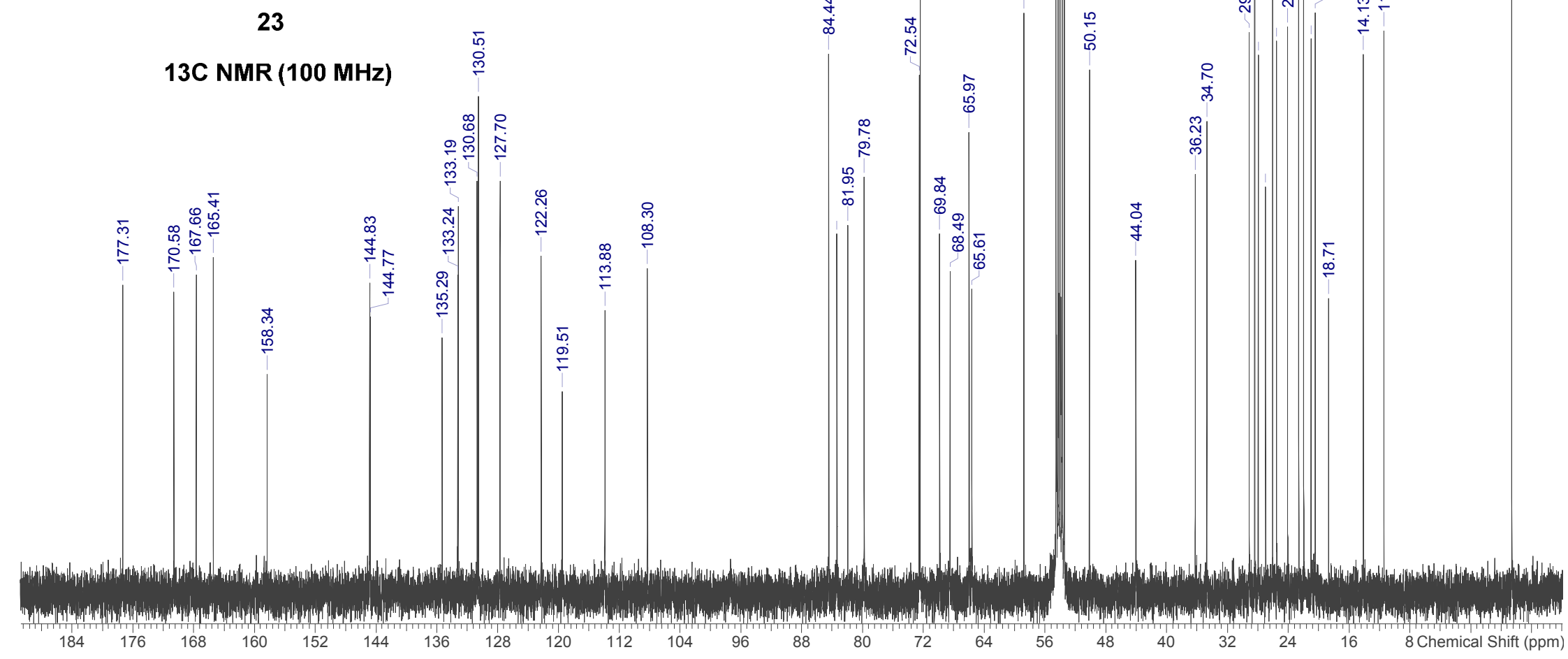




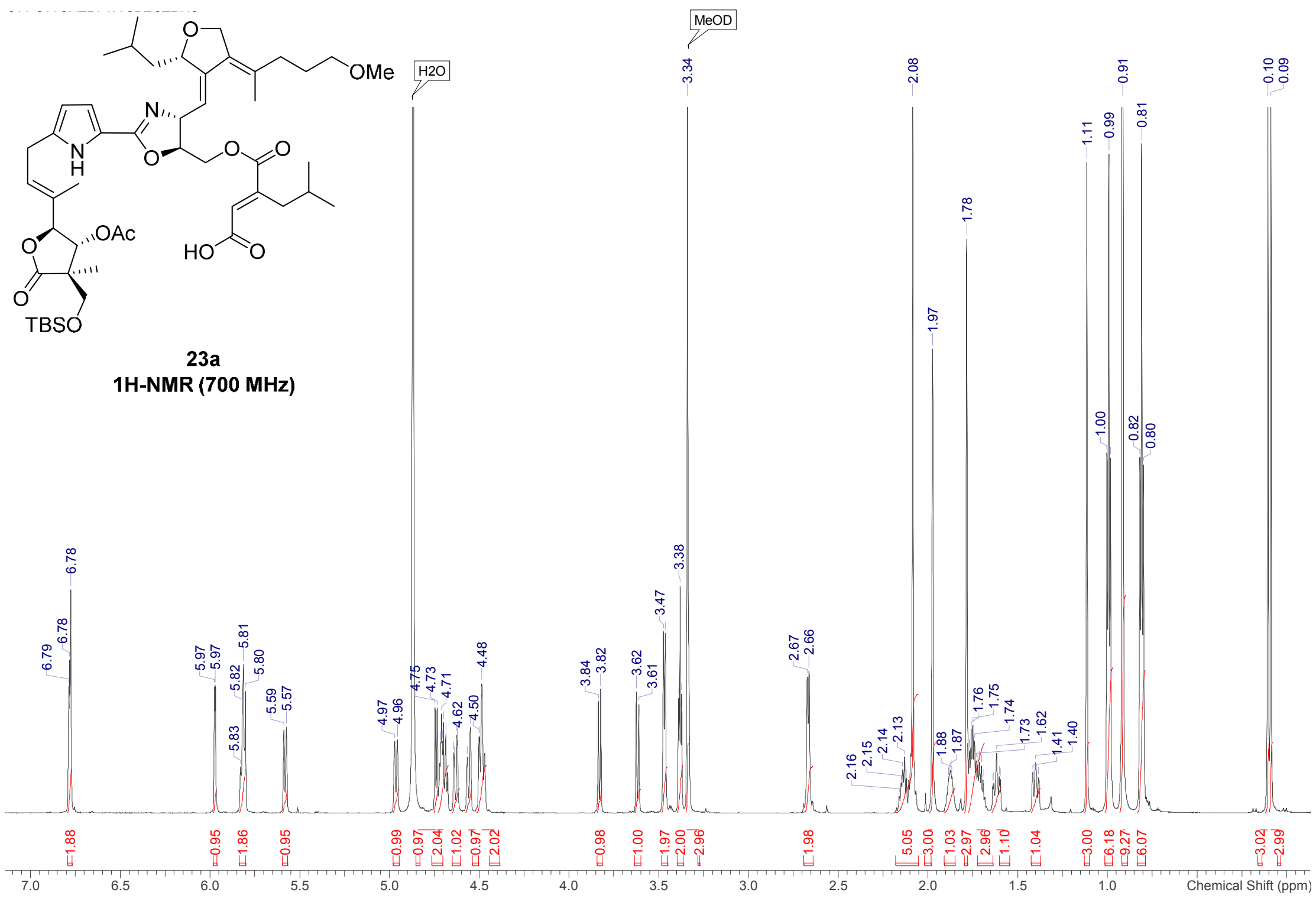



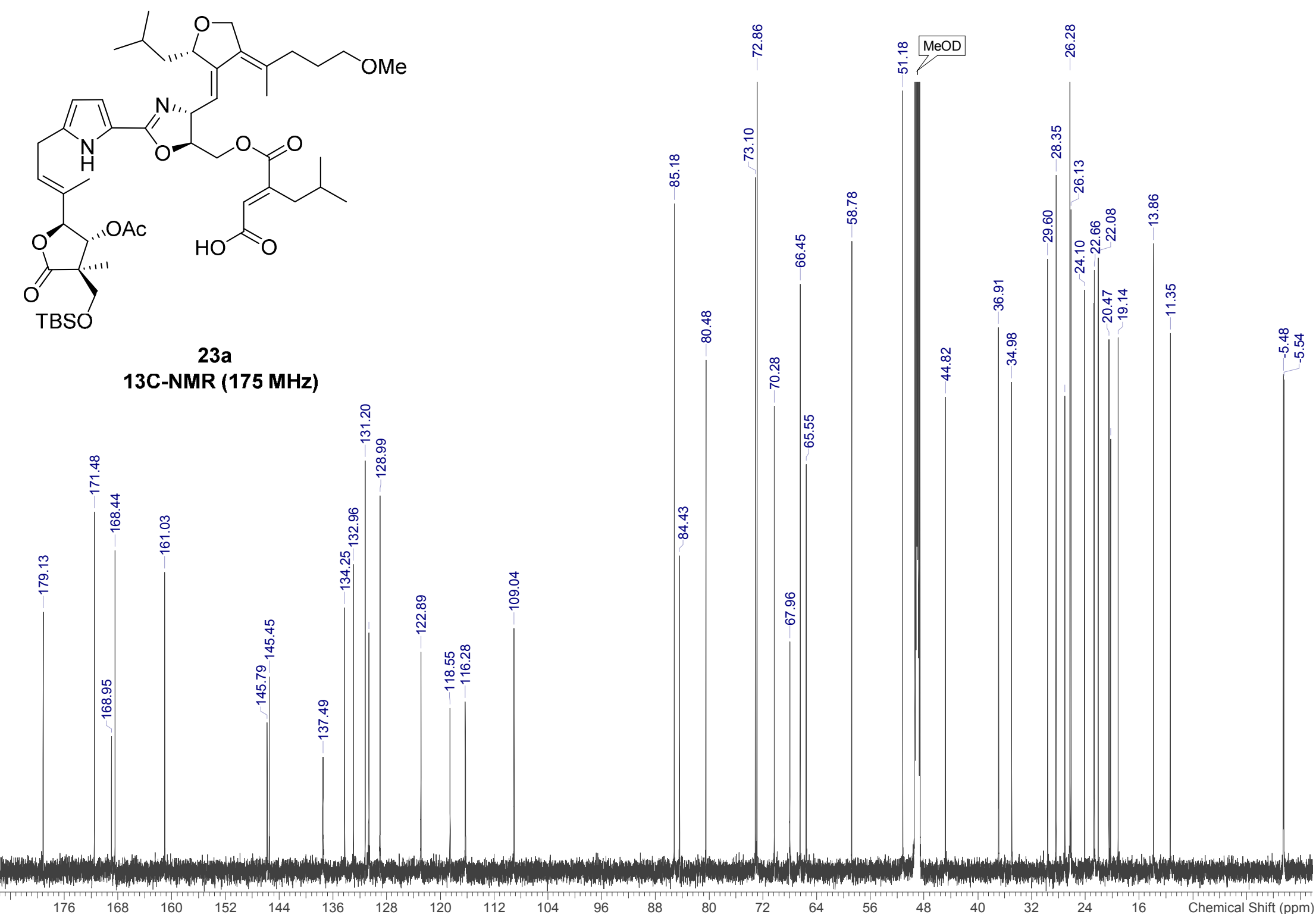


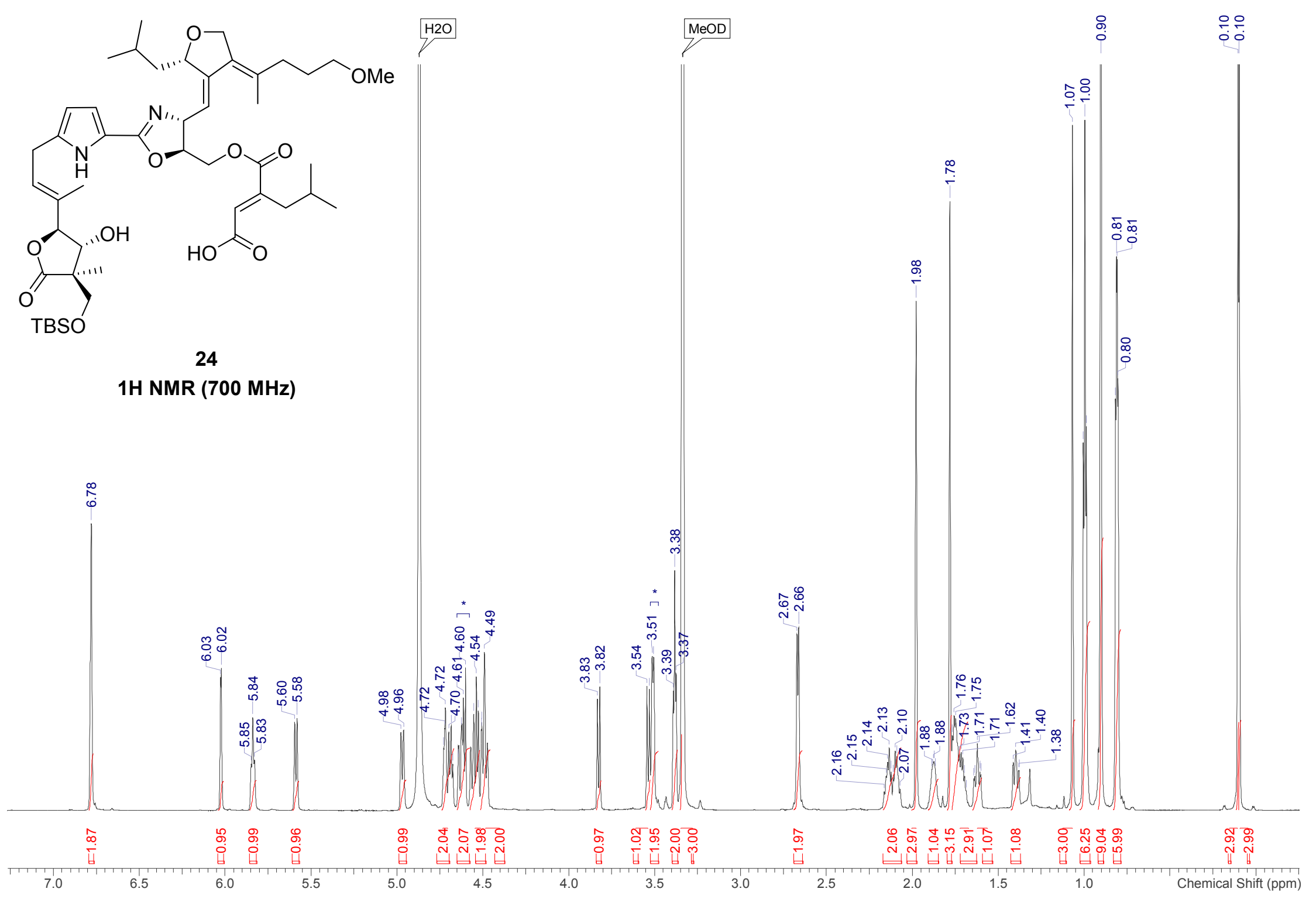



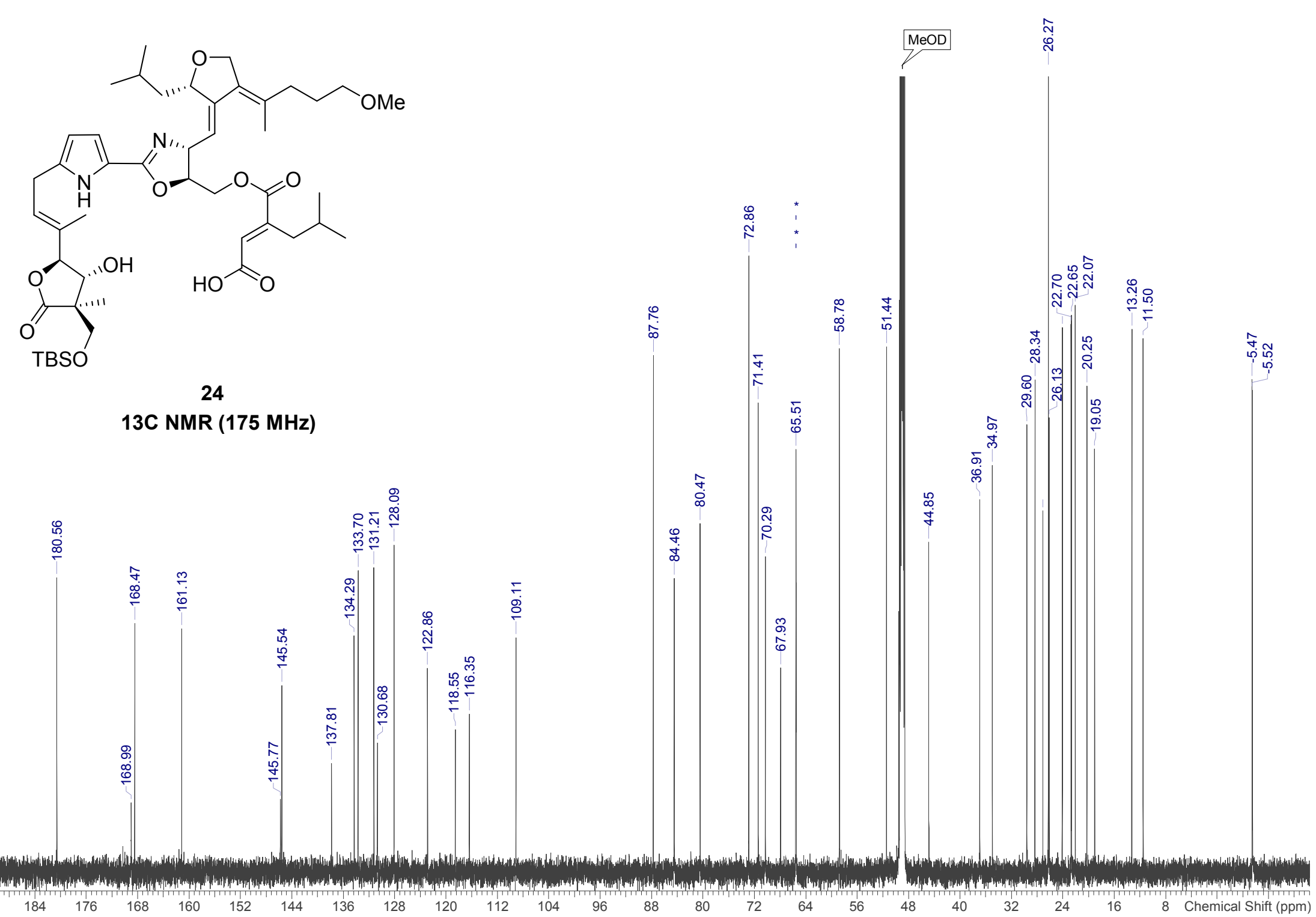


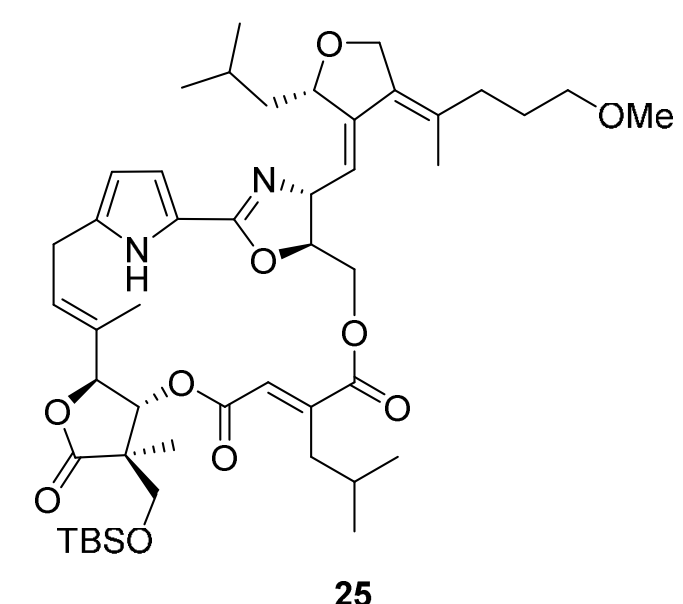

1H-NMR (700 MHz)

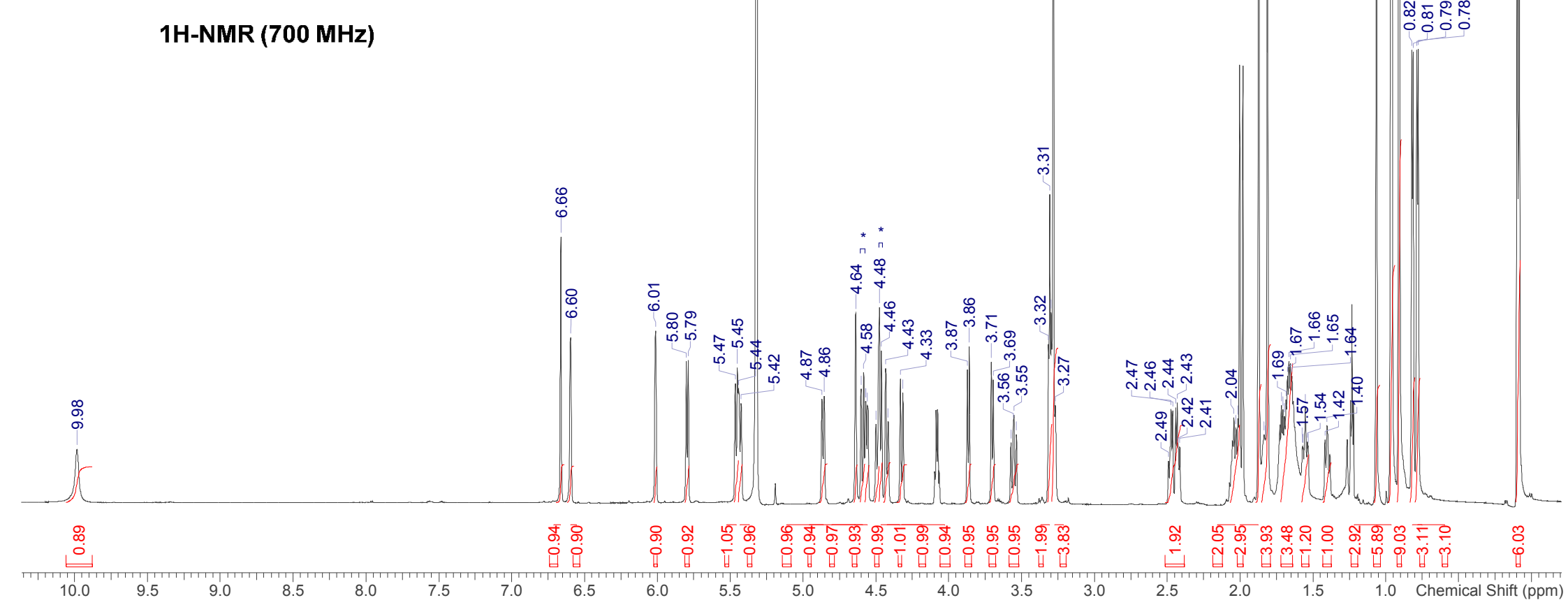



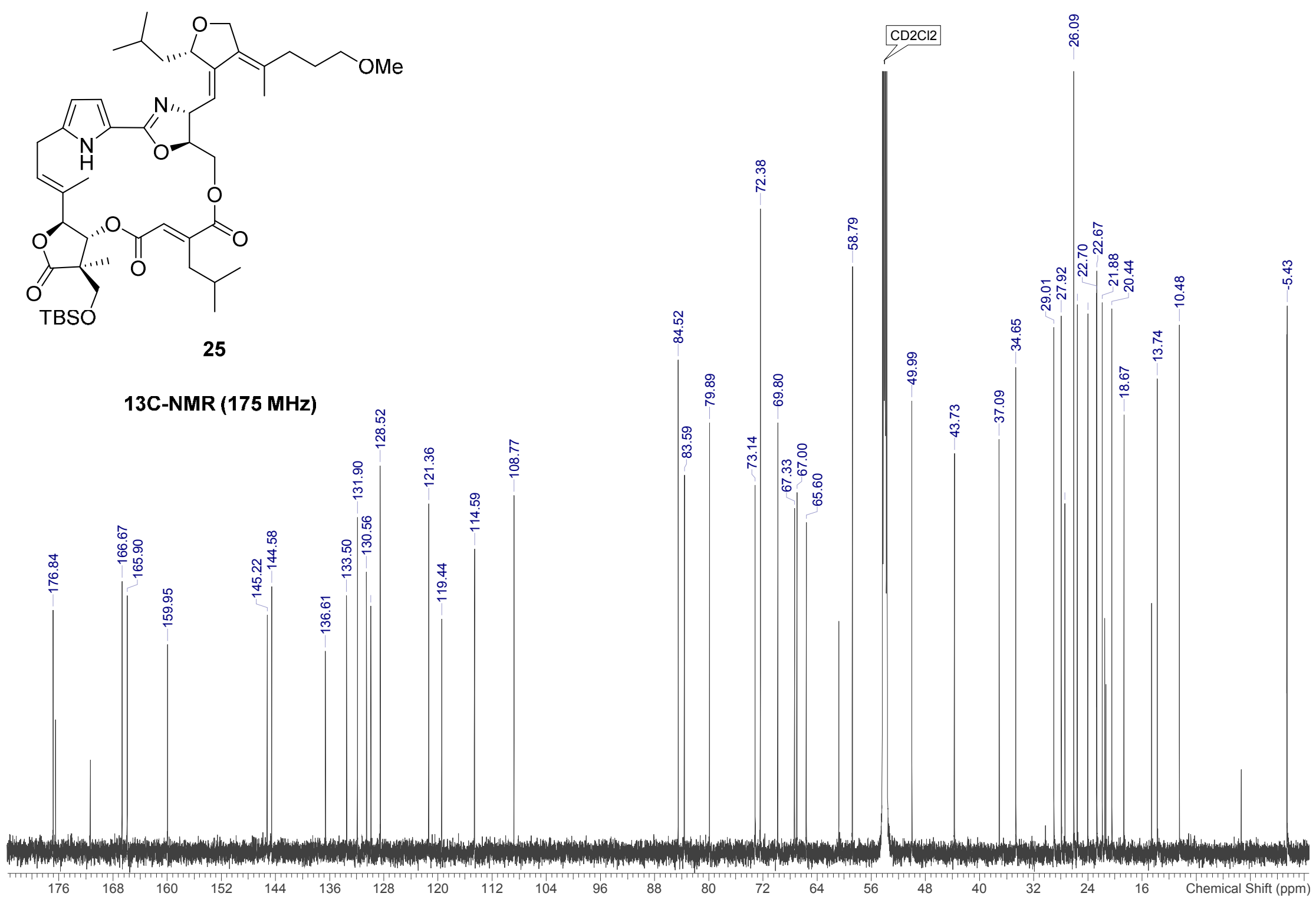


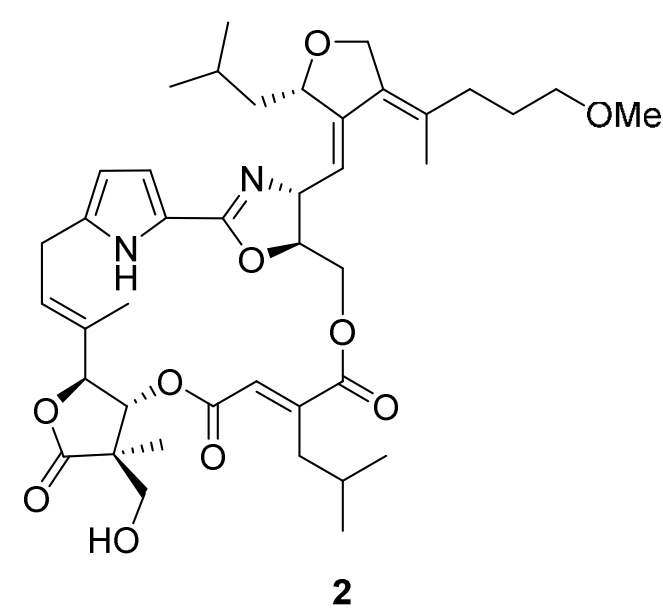

1H-NMR (700 MHz)

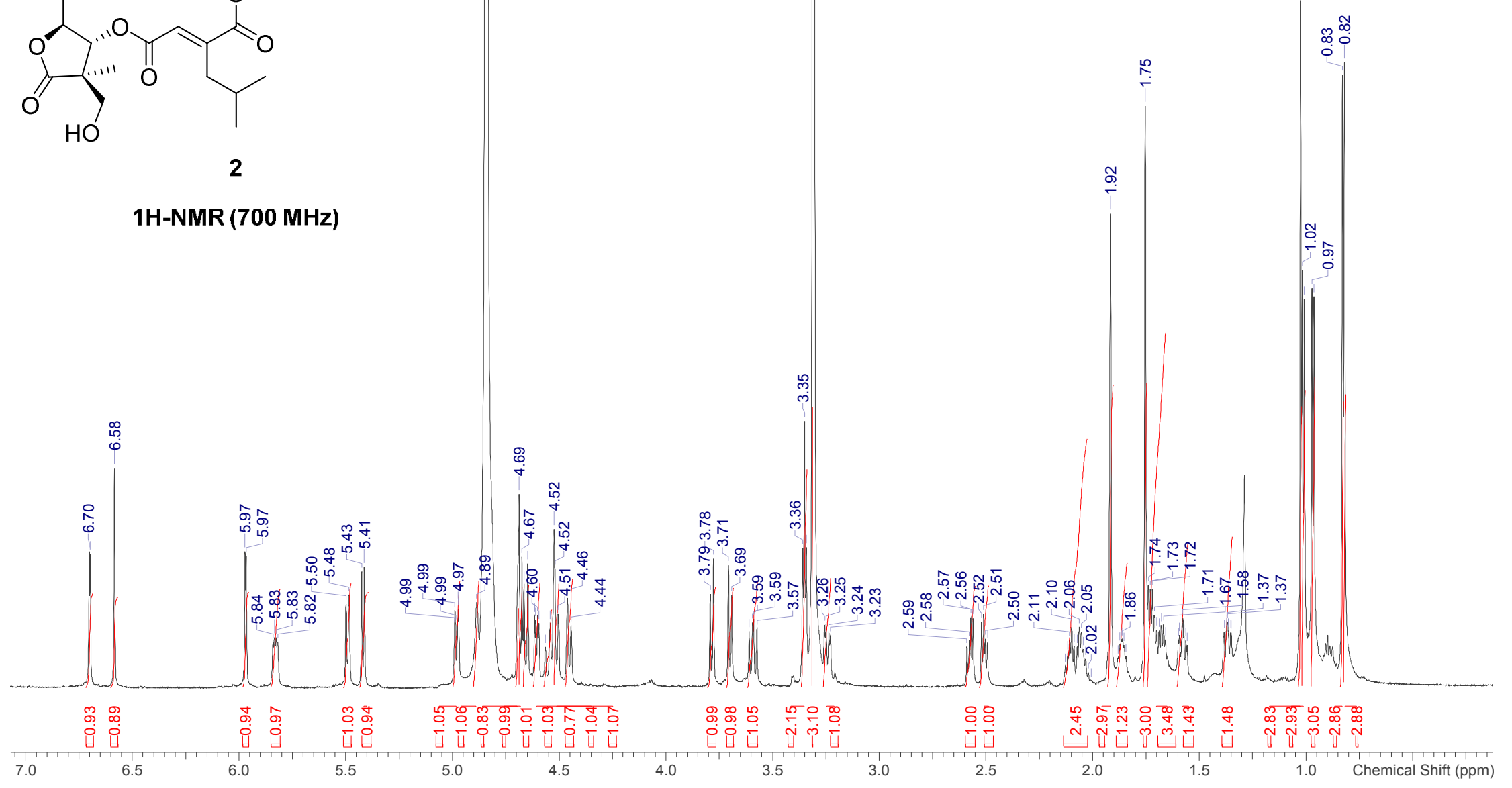

MeOD

$\stackrel{8}{:}$

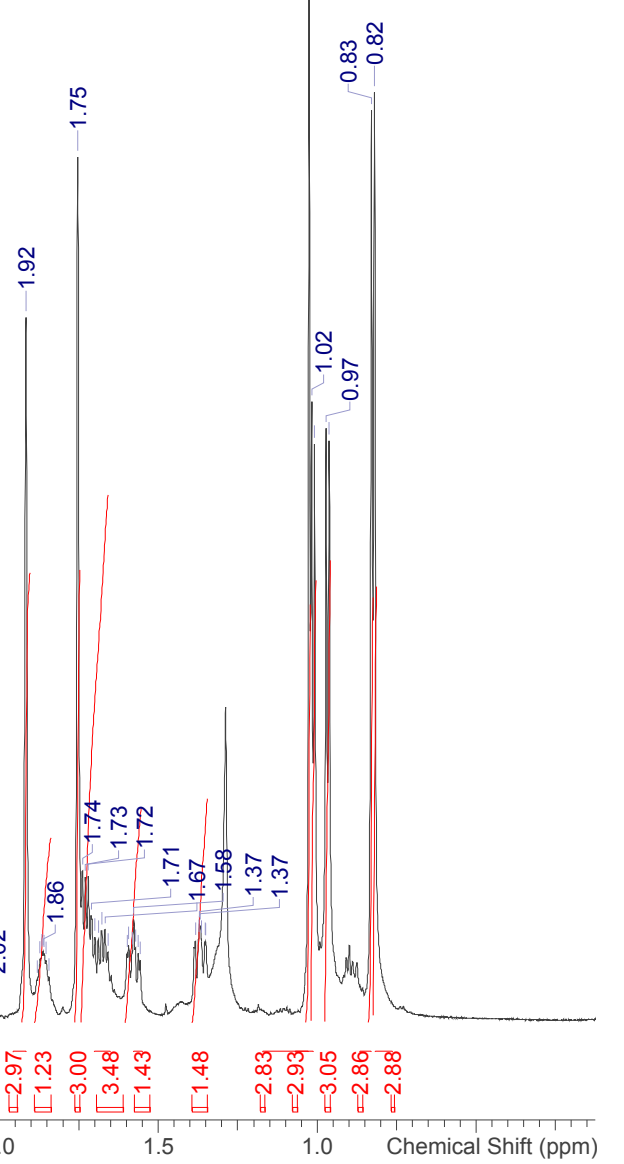




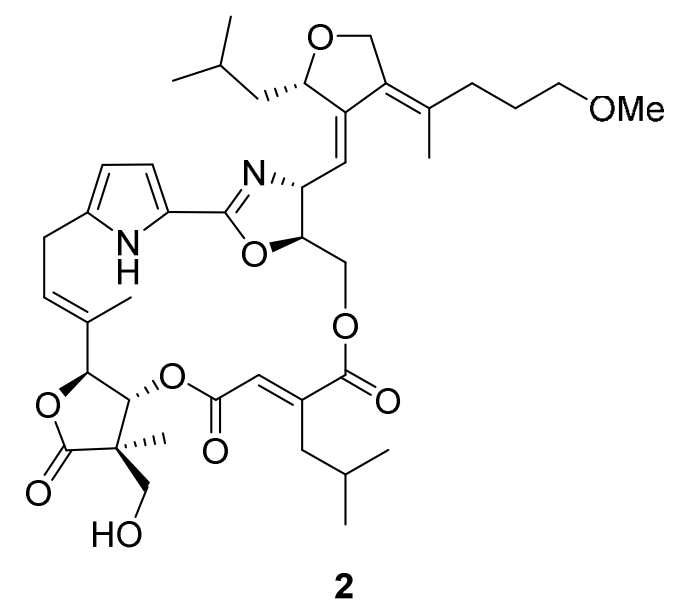

13C-NMR (175 MHz)

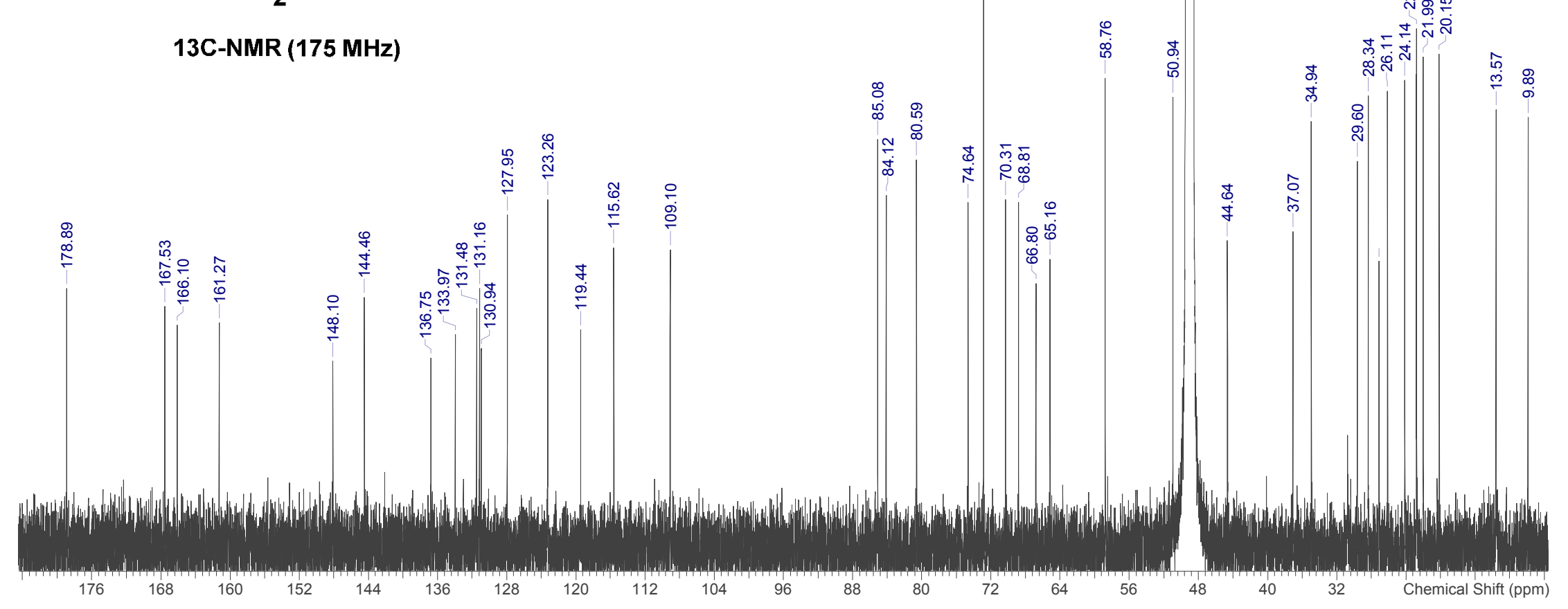




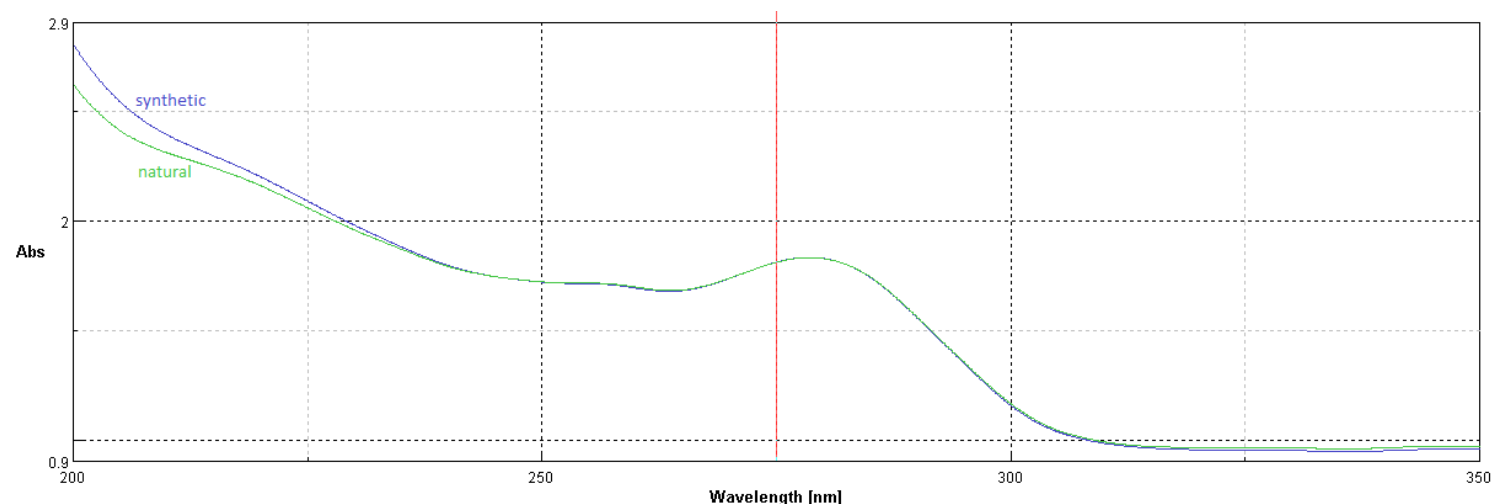

Scheme S 1: CD-Spectra-overlay of synthetic and natural leupyrrin $B_{1}\left(c=10^{-4} \mathrm{~mol} / \mathrm{L}, \mathrm{MeCN}\right)$ 


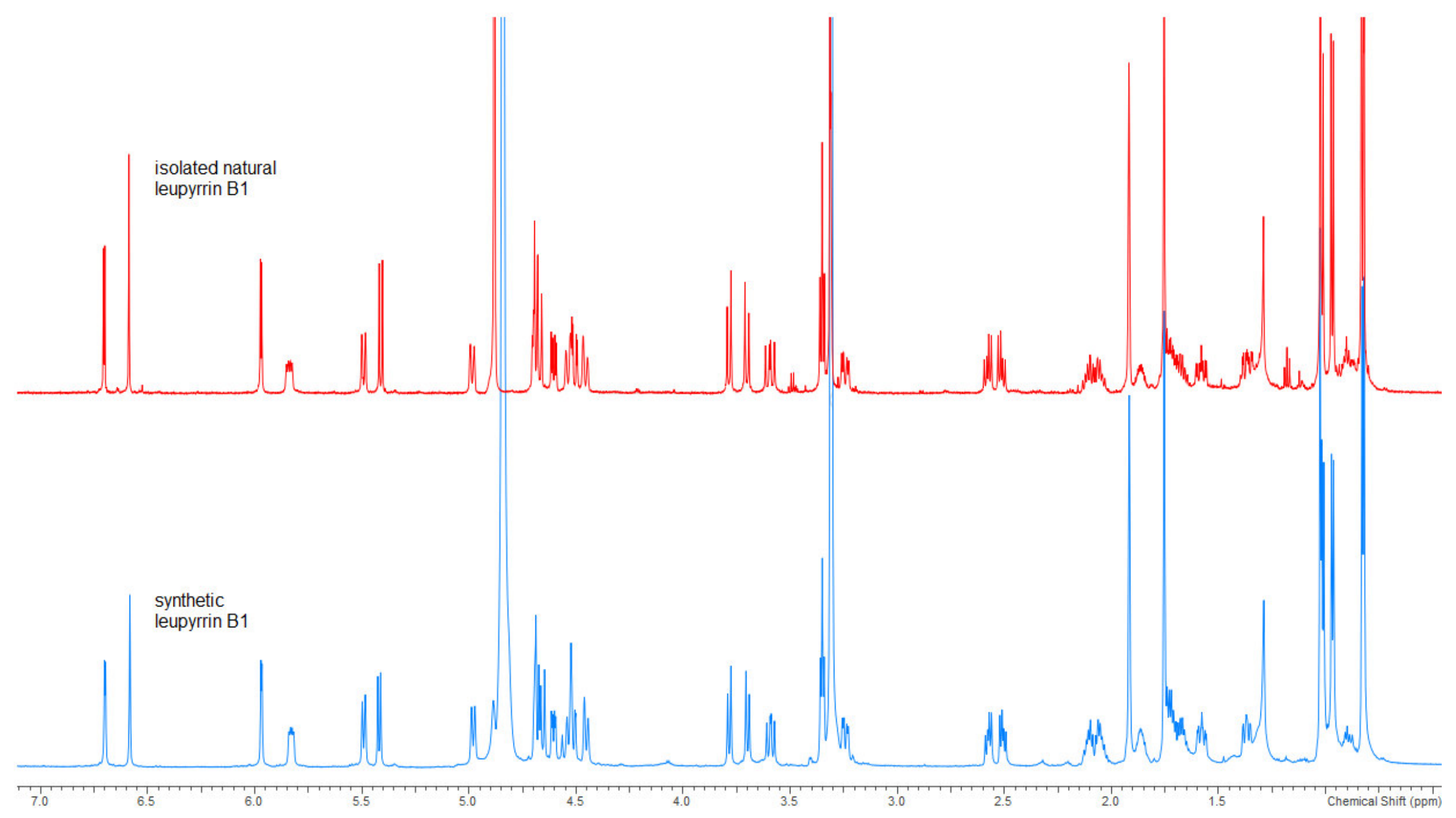




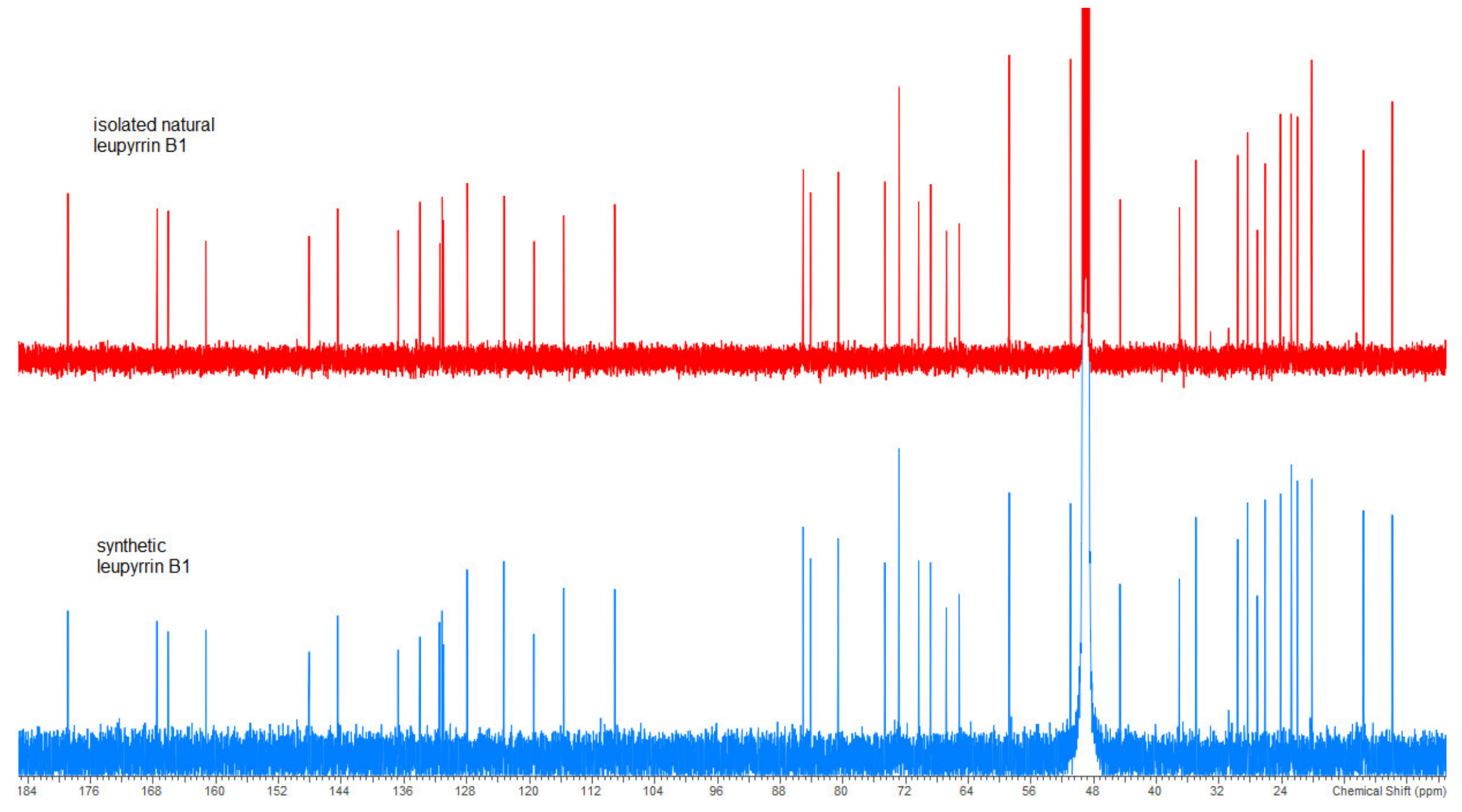




\section{Protease assays.}

The spectrophotometric inhibition assays for five proteases were performed with chromogenic peptide substrates, which, upon cleavage, release para-nitroaniline (pNA). Progress curves were followed over 10 min (HLE, trypsin), $1 \mathrm{~h}$ (cathepsins B and L) or $12.5 \mathrm{~min}$ (chymotrypsin), monitored at $405 \mathrm{~nm}$ and analyzed by linear regression. No inhibition related to more than $95 \%$ activity in duplicate measurements at a single inhibitor concentration $\geq 1 \mu \mathrm{M}$. $\mathrm{IC}_{50}$ values were determined from duplicate measurements by nonlinear regression using equation $\mathrm{v}_{\mathrm{s}}=\mathrm{v}_{0} /\left(1+[\mathrm{I}] / \mathrm{IC}_{50}\right)$, where $\mathrm{v}_{\mathrm{s}}$ is the steady-state rate, $\mathrm{v}_{0}$ is the rate in the absence of the inhibitor, and [I] is the inhibitor concentration. Standard errors of the mean refer to the non-linear regression analysis. HLE was assayed at $25{ }^{\circ} \mathrm{C}$ with MeO-Suc-Ala-Ala-Pro-Val-pNA at a substrate concentration of $100 \mu \mathrm{M}\left(1.85 \times K_{\mathrm{m}}\right){ }^{7}$ A modified assay was applied. ${ }^{8}$ Cathepsins B and L were assayed at $37{ }^{\circ} \mathrm{C}$ with $500 \mu \mathrm{M}\left(0.45 \times K_{\mathrm{m}}\right) \mathrm{Z}$-Arg-Arg-pNA and $100 \mu \mathrm{M}$ Z-PheArg-pNA $\left(5.88 \times K_{\mathrm{m}}\right)$ respectively. ${ }^{9}$ Chymotrypsin was assayed at $25{ }^{\circ} \mathrm{C}$ with $200 \mu \mathrm{M}$ $\left(2.68 \times K_{\mathrm{m}}\right)$ Suc-Ala-Ala-Pro-Phe-pNA. ${ }^{7}$ Trypsin was assayed at $25{ }^{\circ} \mathrm{C}$ with $200 \mu \mathrm{M}$ $\left(2.70 \times K_{\mathrm{m}}\right)$ Suc-Ala-Ala-Pro-Arg-pNA. ${ }^{10}$
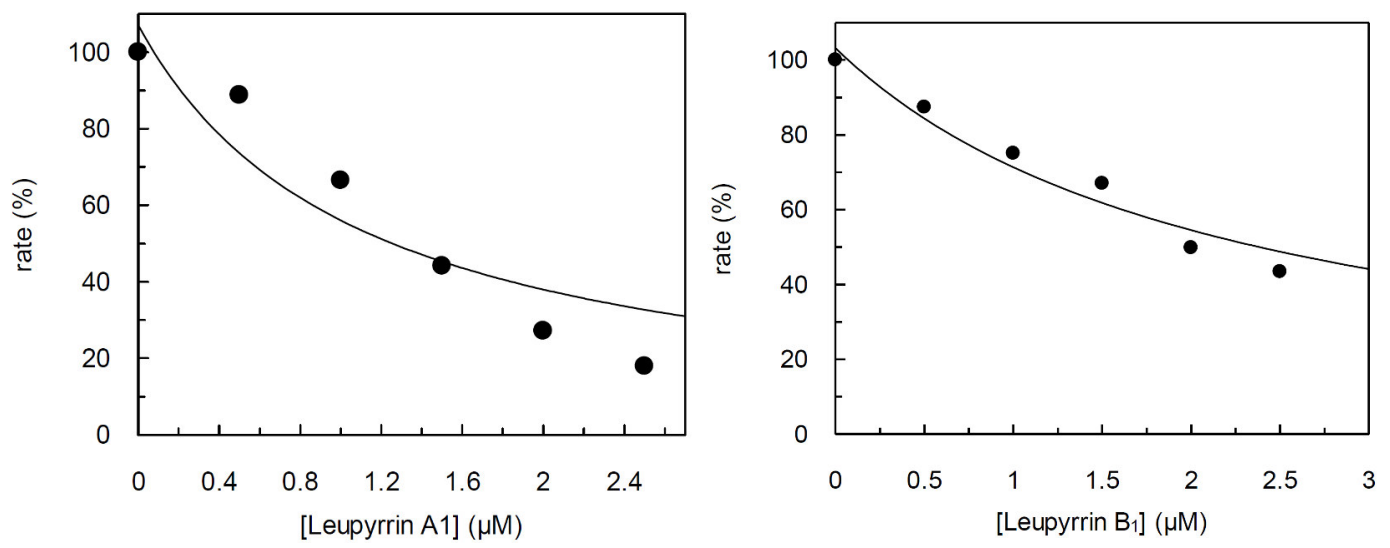

Figure S1. Concentration-dependent inhibition of HLE by leupyrrins $A_{1}$ (left) and $B_{1}$ (right)

\footnotetext{
${ }^{7}$ Gütschow, M.; Pietsch, M.; Themann, A.; Fahrig, J.; Schulze, B. J. Enzyme Inhib. Med. Chem. 2005, 20, 341-347.

${ }^{8}$ Steinebach, C.; Schulz-Fincke, A. C.; Schnakenburg, G.; Gütschow, M. RSC Adv. 2016, 6, 15430-15440.

${ }^{9}$ Frizler, M.; Lohr, F.; Lülsdorff, M.; Gütschow, M. Chem. Eur. J. 2011, 17, 11419-11423.

${ }^{10}$ Sisay, M. T.; Steinmetzer, T.; Stirnberg, M.; Maurer, E.; Hammami, M.; Bajorath, J.; Gütschow, M. J. Med. Chem. 2010, 53, 5523-5535.
} 